\title{
A generalized eigenvalue problem for quasi-orthogonal rational functions
}

\author{
K. Deckers · A. Bultheel · J. Van Deun
}

Received: date / Accepted: date

\begin{abstract}
In general, the zeros of an orthogonal rational function (ORF) on a subset of the real line, with poles among $\left\{\alpha_{1}, \ldots, \alpha_{n}\right\} \subset\left(\mathbb{C}_{0} \cup\{\infty\}\right)$, are not all real (unless $\alpha_{n}$ is real), and hence, they are not suitable to construct a rational Gaussian quadrature rule (RGQ). For this reason, the zeros of a so-called quasiORF or a so-called para-ORF are used instead. These zeros depend on one single parameter $\tau \in(\mathbb{C} \cup\{\infty\})$, which can always be chosen in such a way that the zeros are all real and simple. In this paper we provide a generalized eigenvalue problem to compute the zeros of a quasi-ORF and the corresponding weights in the RGQ. First, we study the connection between quasi-ORFs, paraORFs and ORFs. Next, a condition is given for the parameter $\tau$ so that the zeros are all real and simple. Finally, some illustrative and numerical examples are given.
\end{abstract}

Keywords Quasi-orthogonal rational functions · para-orthogonal rational functions $\cdot$ generalized eigenvalue problem $\cdot$ rational Gaussian quadrature

Mathematics Subject Classification (2000) 42C05 - 65D32 $\cdot 65 \mathrm{~F} 15$

This work is partially supported by the Belgian Network DYSCO (Dynamical Systems, Control, and Optimization), funded by the Interuniversity Attraction Poles Programme, initiated by the Belgian State, Science Policy Office. The scientific responsibility rests with its authors.

The first author is a Postdoctoral Fellow of the Research Foundation - Flanders (FWO).

K. Deckers

Department of Computer Science, Katholieke Universiteit Leuven, Heverlee, Belgium.

E-mail: karl.deckers@cs.kuleuven.be

A. Bultheel

Department of Computer Science, Katholieke Universiteit Leuven, Heverlee, Belgium.

E-mail: adhemar.bultheel@cs.kuleuven.be

J. Van Deun

Department of Mathematics and Computer Science, University of Antwerp, Antwerp,

Belgium.

E-mail: joris.vandeun@ua.ac.be 


\section{Introduction}

Since the fundamental work of Stieltjes and Chebyshev, among others, in the $19^{\text {th }}$ century, orthogonal polynomials (OPs) have been an essential tool in the analysis of basic problems in mathematics and engineering. For example, moment problems, numerical quadrature, rational and polynomial approximation and interpolation, linear algebra, and all the direct or indirect applications of these techniques in engineering are all indebted to the basic properties of OPs. Mostly orthogonality has been considered on the complex unit circle or on (a subset of) the real line.

Orthogonal rational functions (ORFs) were first introduced by Džrbašian in the 1960s. Most of his papers appeared in Russian literature, but an accessible survey in English can be found in $[19,25]$. These ORFs are a generalization of OPs in such a way that they are of increasing degree with a given sequence of poles, and the OPs result if all the poles are at infinity. During the last years, many classical results of OPs have been extended to the case of ORFs.

Several generalizations for ORFs on the complex unit circle and the whole real line have been gathered in book [6, Chapt. 2-10] (e.g., the recurrence relation and the Favard theorem, the Christoffel-Darboux relation, properties of the zeros, etc.). Further, we refer to $[4,5,9]$ and to [41] for the use of these ORFs in respectively numerical quadrature and system identification, while several results about matrix-valued ORFs can be found in e.g., [20,21].

Of course, many of the classical OPs are not defined with respect to a measure on the whole unit circle or the whole real line. Several theoretical results for ORFs on a subset of the real line can be found in e.g., [6, Chapt. 11]. For the special case in which this subset is a real half-line or an interval, we refer to $[7,8]$ and $[16,33]$ respectively, while some computational aspects have been dealt with in e.g., $[17,34-36,38]$.

Under some mild conditions, ORFs on a subset of the real line satisfy a three-term recurrence relation (see [6, Chapt. 11.1] and [15]), and hence, the zeros of these ORFs are eigenvalues of a generalized eigenvalue problem (GEP) obtained by this three-term recurrence relation (see e.g., [32]). In general, however, the zeros of ORFs are not all real so that they are not suitable to construct a rational Gaussian quadrature rule (see Lemma 12 further on). For this reason, the zeros of a so-called quasi-orthogonal rational function (qORF) or a so-called para-orthogonal rational function (pORF) are used instead. The zeros of a qORF or pORF depend on one single parameter $\tau$, which can always be chosen in such a way that the zeros are all real and simple (see [6, Chapt. $11.5]$ and [37]).

The qORFs under consideration are rational generalizations of the quasiorthogonal polynomials, introduced by Riesz in [26], and later redefined in a more general way by Chihara in [11]. In contrast, although the definition of the pORFs in this paper is inspired by the definition of the well-known paraorthogonal polynomials on the unit circle, introduced by Jones et. al. in [24] 
(see also e.g., [10,23,27-29]), the former are no rational generalizations of the latter. ${ }^{1}$

The aim of this paper is to present a GEP to compute the zeros of a qORF or pORF (and, if necessary, the corresponding weights in the rational Gaussian quadrature rule). The possible applications of these zeros involves every application where polynomials are used to interpolate a function $f$ on a subset of the real line. In this respect, the main advantage of rational interpolation is that convergence can be sped up by taking the singularities of the function $f$ into account, when the interpolants (based on polynomial interpolation) converge too slowly to the function $f$ for increasing number of interpolation points. Similar as in the polynomial case, interpolating in the nodes of a rational Gaussian quadrature rule has nice properties regarding convergence and numerical stability. See e.g., $[22,31,33,37,39,43])$ and $[1-3$, $30,42]$, for their usefulness in numerical integration and in numerically solving differential equations using rational spectral methods respectively.

The outline is as follows: After giving the necessary theoretical background in Section 2, in Section 3 we study the relation between qORFs and ORFs, and present a GEP to compute the zeros of a qORF. Next, in Section 4 we study the relation between qORFs and pORFs. Then in Section 5 we recall the use of qORFs in rational Gaussian quadrature and give conditions so that their zeros are all real and simple. Finally, some illustrative and numerical examples are given in Sections 6-7 for the case in which orthogonality is with respect to the Chebyshev weight functions $(1-x)^{a}(1+x)^{b}$ on the interval $[-1,1]$, where $a, b \in\left\{ \pm \frac{1}{2}\right\}$.

\section{Preliminaries}

The field of complex numbers will be denoted by $\mathbb{C}$ and the Riemann sphere by $\overline{\mathbb{C}}=\mathbb{C} \cup\{\infty\}$. For the real line we use the symbol $\mathbb{R}$ and for the extended real line $\overline{\mathbb{R}}=\mathbb{R} \cup\{\infty\}$. Further, the positive half line will be represented by $\mathbb{R}^{+}=\{x \in \mathbb{R}: x \geq 0\}$. Let $a \in \mathbb{C}$, then $\Re\{a\}$ refers to the real part of $a$, while $\Im\{a\}$ refers to the imaginary part, and the imaginary unit will be denoted by i. For $a \neq 0$ we will also use the short notation $\mathfrak{T}\{a\}$, defined by

$$
\mathfrak{T}\{a\}=\Im\{1 / \bar{a}\}= \begin{cases}\frac{\Im\{a\}}{|a|^{2}}, & a \in \mathbb{C} \\ 0, & a=\infty .\end{cases}
$$

The unit circle and the open unit disc are denoted respectively by

$$
\mathbb{T}=\{z \in \mathbb{C}:|z|=1\} \quad \text { and } \quad \mathbb{D}=\{z \in \mathbb{C}:|z|<1\} .
$$

Whenever the value zero is omitted in a set $X \subseteq \overline{\mathbb{C}}$, this will be represented by $X_{0}$. Similarly, the complement of a set $Y \subset \overline{\mathbb{C}}$ with respect to a set $X \subseteq \overline{\mathbb{C}}$ will be given by $X_{Y}$, i.e., $X_{Y}=\{t \in X: t \notin Y\}$.

\footnotetext{
1 For a rational generalization of para-orthogonal polynomials on the unit circle, see e.g., [6, Chapt. 5].
} 
For any complex function $f$, we define the involution operation or super-c conjugate by $f^{c}(x)=\overline{f(\bar{x})}$. With $\mathcal{P}_{k}$ we denote the space of polynomials of degree less than or equal to $k$, while $\mathcal{P}$ represents the space of all polynomials. Further, we will use the short notation $[f(x)]_{x=a}$ to denote $\lim _{x \rightarrow a} f(x)$.

Let there be fixed a sequence of poles $\mathcal{A}=\left\{\alpha_{1}, \alpha_{2}, \ldots\right\} \subset \overline{\mathbb{C}}_{0}$, where the poles are arbitrary complex or infinite; hence, they do not have to appear in pairs of complex conjugates. The rational functions we then deal with, are of the form

$$
f_{k}(x)=\frac{c_{k} x^{k}+c_{k-1} x^{k-1}+\cdots+c_{0} x^{0}}{\left(1-x / \alpha_{1}\right)\left(1-x / \alpha_{2}\right) \cdots\left(1-x / \alpha_{k}\right)}, \quad k=1,2, \ldots .
$$

Note that, whenever $\alpha_{j}=\infty$ for every $j \geqslant 1$, the "rational functions" $f_{k}(x)$ in (1) are in fact polynomials of degree $k$. Thus the polynomial case is automatically accounted for.

Define the factors

$$
Z_{k}(x):=Z_{\alpha_{k}}(x)=\frac{x}{1-x / \alpha_{k}}, \quad k=1,2, \ldots,
$$

and the basis functions

$$
b_{0}(x) \equiv 1, \quad b_{k}(x)=b_{k-1}(x) Z_{k}(x), \quad k=1,2, \ldots .
$$

These basis functions generate the nested spaces of rational functions with poles in $\mathcal{A}$ defined by

$\mathcal{L}_{-1}=\{0\}, \quad \mathcal{L}_{0}=\mathbb{C}, \quad \mathcal{L}_{k}:=\mathcal{L}\left\{\alpha_{1}, \ldots, \alpha_{k}\right\}=\operatorname{span}\left\{b_{0}, \ldots, b_{k}\right\}, k=1,2, \ldots$, and $\mathcal{L}=\bigcup_{k=0}^{\infty} \mathcal{L}_{k}$. Let

$$
\pi_{0}(x) \equiv 1, \quad \pi_{k}(x)=\prod_{j=1}^{k}\left(1-x / \alpha_{j}\right), \quad k=1,2, \ldots,
$$

then for $k \geqslant 0$ we may write equivalently

$$
b_{k}(x)=\frac{x^{k}}{\pi_{k}(x)} \quad \text { and } \quad \mathcal{L}_{k}=\left\{p_{k} / \pi_{k}: p_{k} \in \mathcal{P}_{k}\right\}, \quad k=0,1,2, \ldots .
$$

In the remainder we will also use the short notations $\mathcal{L}_{k}^{[\alpha]}:=\mathcal{L}\left\{\alpha_{1}, \ldots, \alpha_{k-1}, \alpha\right\}$ (i.e., the space of rational functions with the same dimension as $\mathcal{L}_{k}$, but with the last pole $\alpha_{k}$ replaced by the pole $\left.\alpha\right)$ and $\mathcal{L}_{k}(\alpha):=\left\{(1-\cdot / \alpha) p_{k-1}(\cdot) / \pi_{k}(\cdot)\right.$ : $\left.p_{k-1} \in \mathcal{P}_{k-1}\right\}=\frac{Z_{k}}{Z_{\alpha}} \mathcal{L}_{k-1}$.

Note that $\mathcal{L}_{k}$ and $\mathcal{L}$ are rational generalizations of $\mathcal{P}_{k}$ and $\mathcal{P}$. Indeed, if $\alpha_{j}=\infty$ for every $j \geqslant 1$, the expression in (2) becomes $Z_{k}(x)=x$ and the expression in (3) becomes $b_{k}(x)=x^{k}$. With the definition of the super-c conjugate we introduce $\mathcal{L}_{k}^{c}=\left\{f^{c}: f \in \mathcal{L}_{k}\right\}$.

The superstar transformation of a complex function $f_{k} \in \mathcal{L}_{k} \backslash \mathcal{L}_{k-1}$ is defined as

$$
f_{k}^{*}(x)=\frac{b_{k}(x)}{b_{k}^{c}(x)} f_{k}^{c}(x) .
$$


Note that the factor $\frac{b_{k}}{b_{k}^{c}}$ merely replaces the polynomial with zeros $\left\{\bar{\alpha}_{j}\right\}_{j=1}^{k}$ in the denominator of $f_{k}^{c}(x)$ by a polynomial with zeros $\left\{\alpha_{j}\right\}_{j=1}^{k}$ so that $\mathcal{L}_{k}^{*}:=$ $\left\{f^{*}: f \in \mathcal{L}_{k}\right\}=\mathcal{L}_{k}$.

Consider an inner product defined by a linear functional $\mathfrak{F}$ :

$$
\langle f, g\rangle_{\mathfrak{F}}=\mathfrak{F}\left\{f g^{c}\right\}, \quad f, g \in \mathcal{L},
$$

where the linear functional $\mathfrak{F}$ is assumed to be hermitian positive-definite (HPD), i.e.,

$$
\mathfrak{F}\left\{f g^{c}\right\}=\overline{\mathfrak{F}\left\{f^{c} g\right\}} \quad \text { and } \quad \mathfrak{F}\left\{f f^{c}\right\}>0 \text { for } f \neq 0 .
$$

Orthogonalizing the basis functions $\left\{b_{0}, b_{1}, \ldots\right\}$ with respect to this inner product, we obtain a sequence of orthogonal rational functions (ORFs) $\left\{\phi_{0}, \phi_{1}, \ldots\right\}$, with $\phi_{k} \in \mathcal{L}_{k} \backslash \mathcal{L}_{k-1}$, so that $\phi_{k} \perp_{\mathfrak{F}} \mathcal{L}_{k-1}$, i.e.,

$$
\left\langle\phi_{k}, \phi_{j}\right\rangle_{\mathfrak{F}}=\frac{1}{\left|d_{k}\right|^{2}} \delta_{k, j}, \quad d_{k} \in \mathbb{C}_{0}, k, j=0,1, \ldots,
$$

where $\delta_{k, j}$ is the Kronecker Delta. In the special case in which $\varphi_{k}(x)=$ $d_{k} \phi_{k}(x)$, so that

$$
\left\|\varphi_{k}\right\|_{\mathfrak{F}}:=\sqrt{\left\langle\varphi_{k}, \varphi_{k}\right\rangle_{\mathfrak{F}}}=1
$$

we say that $\varphi_{k}$ is an orthonormal rational function (nORF).

Put by convention $\alpha_{-1}=\alpha_{0}=\infty$. We then call a rational function $f_{k}=\frac{p_{k}}{\pi_{k}} \in \mathcal{L}_{k} \backslash \mathcal{L}_{k-1}$, with $k \geqslant 0$, exceptional (respectively degenerate) iff $p_{k}\left(\alpha_{k-1}\right)=0$ (respectively $\left.p_{k}\left(\bar{\alpha}_{k-1}\right)=0\right)$. A zero of $p_{k}$ at $\infty$ means that the degree of $p_{k}$ is less than $k$. Further, for $k>1$ we say that $f_{k}$ is singular iff $p_{k}\left(\alpha_{j}\right)=0$ for at least one $j \in\{0, \ldots, k-2\}$. Finally, $f_{k}$ is called regular iff $f_{k}$ is not singular, not degenerate and not exceptional. With these definitions we now introduce the following notations:

$$
\begin{aligned}
{ }^{e} \mathcal{L}_{k} & :=\left\{f \in \mathcal{L}_{k} \backslash \mathcal{L}_{k-1}: f \text { is not exceptional }\right\}, \\
{ }^{d} \mathcal{L}_{k} & :=\left\{f \in{ }^{e} \mathcal{L}_{k}: f \text { is not degenerate }\right\} \\
{ }^{r} \mathcal{L}_{k} & :=\left\{f \in \mathcal{L}_{k} \backslash \mathcal{L}_{k-1}: f \text { is regular }\right\} .
\end{aligned}
$$

In $[15$, Sec. 3] the following three-term recurrence relation has been proved for nORFs $\varphi_{k} \in \mathcal{L}_{k} \backslash \mathcal{L}_{k-1}$.

Theorem 1 Consider the nORFs $\varphi_{j} \in \mathcal{L}_{j} \backslash \mathcal{L}_{j-1}$, with $j=k, k-1, k-2$ and $k>0$, and assume $\varphi_{k} \in{ }^{e} \mathcal{L}_{k}, \varphi_{k-1} \in{ }^{d} \mathcal{L}_{k-1}$ and $\varphi_{k-2}$ is not degenerate. Then these nORFs satisfy a three-term recurrence relation of the form

$$
\begin{aligned}
\varphi_{k}(x) & =E_{k} Z_{k}(x)\left\{\left[1+\frac{D_{k}}{Z_{k-1}(x)}\right] \varphi_{k-1}(x)-\frac{C_{k}}{Z_{k-2}^{c}(x)} \varphi_{k-2}(x)\right\} \\
& =E_{k} \psi_{k}(x),
\end{aligned}
$$


where $E_{k} \in \mathbb{C}_{0}, D_{k} \in \mathbb{C}$ and

$$
C_{k}=\frac{1-D_{k} / Z_{k-1}^{c}\left(\alpha_{k-1}\right)}{\bar{E}_{k-1}} \in \mathbb{C}_{0},
$$

with initial conditions $\alpha_{-1}=\alpha_{0}=\infty, \varphi_{-1}(x) \equiv 0$, and $\varphi_{0}(x) \equiv \eta_{0}\|1\|_{\mathfrak{F}}^{-1}=$ $E_{0}$, where $\eta_{0} \in \mathbb{T}$.

The coefficients $E_{k}$ and $D_{k}$ can be expressed in terms of inner products as follows (see [15, Thm. 3.7 and Thm. 3.9]):

$$
E_{k}=\eta_{k}\left\|\psi_{k}\right\|_{\mathfrak{F}}^{-1}, \quad \eta_{k} \in \mathbb{T},
$$

and

$$
D_{k}=\frac{K_{k, j}-L_{k, j}}{\frac{L_{k, j}}{Z_{k-1}\left(\alpha_{k}\right)}+\frac{K_{k, j}}{Z_{k-1}^{c}\left(\alpha_{k-1}\right)}+\delta_{k-1, j} \bar{E}_{k-1}}, \quad j<k,
$$

with

$K_{k, j}=\frac{1}{Z_{k-2}^{c}\left(\alpha_{k}\right)}\left\langle Z_{k} \varphi_{k-2}, \varphi_{j}\right\rangle_{\mathfrak{F}}+\delta_{k-2, j} \quad$ and $\quad L_{k, j}=\bar{E}_{k-1}\left\langle Z_{k} \varphi_{k-1}, \varphi_{j}\right\rangle_{\mathfrak{F}}$.

Whenever $\alpha_{k} \notin \overline{\mathbb{R}}$, the modulus of the coefficient $E_{k}$ can also be computed as follows:

$\left|E_{k}\right|^{2}=\frac{\mathfrak{T}\left\{\alpha_{k}\right\}\left|E_{k-1}\right|^{2}}{\left(\Im\left\{D_{k}\right\}-\left|D_{k}\right|^{2} \mathfrak{T}\left\{\alpha_{k-1}\right\}\right) \cdot\left(\left|E_{k-1}\right|^{2}-4 \mathfrak{T}\left\{\alpha_{k-1}\right\} \cdot \mathfrak{T}\left\{\alpha_{k-2}\right\}\right)+\mathfrak{T}\left\{\alpha_{k-2}\right\}}$.

Finally, the coefficients $E_{k}$ and $D_{k}$ can also be expressed in terms of nORFs $\varphi_{k}$ as follows (see [15, Thm. 5.1]):

$E_{k}=\left[\frac{\varphi_{k}(x)}{Z_{k}(x) \varphi_{k-1}(x)}\right]_{x=\alpha_{k-1}}$ and $D_{k}=\left[\frac{Z_{k-1}(x) \varphi_{k}(x)}{E_{k} Z_{k}(x) \varphi_{k-1}(x)}-Z_{k-1}(x)\right]_{x=\bar{\alpha}_{k-2}}$.

In the opposite direction as in Theorem 1, the following Favard-type theorem has been proved in [14].

Theorem 2 Let $\left\{\chi_{k}\right\}_{k=0}^{\infty}$ be a sequence of rational functions in $\mathcal{L}$, and assume that the following conditions are satisfied:

(A1) $\alpha_{-1}=\alpha_{0}=\infty$ and $\alpha_{k} \in \overline{\mathbb{C}}_{0}, k=1,2, \ldots$;

(A2) $\chi_{k}$ is generated by the three-term recurrence relation (5);

(A3) $\chi_{k} \in \mathcal{L}_{k} \backslash \mathcal{L}_{k-1}, k=0,1,2, \ldots$, and $\chi_{-1} \equiv 0$;

(A4) $D_{k} \in \mathbb{C}$ and $E_{k} \in \mathbb{C}_{0}, k=1,2, \ldots$;

$$
\Im\left\{D_{k}\right\}=\frac{\mathfrak{T}\left\{\alpha_{k}\right\}}{\left|E_{k}\right|^{2}}-\frac{\mathfrak{T}\left\{\alpha_{k-2}\right\}}{\left|E_{k-1}\right|^{2}}
$$

if $\alpha_{k-1} \in \overline{\mathbb{R}}_{0}$, respectively

$$
\Re\left\{D_{k}\right\}^{2}+\left\{\Im\left\{D_{k}\right\}-\mathbf{i} Z_{k-1}\left(\bar{\alpha}_{k-1}\right)\right\}^{2}=-\left\{Z_{k-1}\left(\bar{\alpha}_{k-1}\right)\right\}^{2} \frac{\left|E_{k-1}\right|^{2}}{\left|E_{k}\right|^{2}} \cdot \frac{\Delta_{k}}{\Delta_{k-1}}
$$


if $\alpha_{k-1} \notin \overline{\mathbb{R}}$, where $\Delta_{k}=\left|E_{k}\right|^{2}-4 \mathfrak{T}\left\{\alpha_{k}\right\} \cdot \mathfrak{T}\left\{\alpha_{k-1}\right\}>0, k=1,2, \ldots$, with $E_{0} \in \mathbb{C}_{0}$.

(A6) $C_{k}=\frac{1-D_{k} / Z_{k-1}^{c}\left(\alpha_{k-1}\right)}{\bar{E}_{k-1}} \in \mathbb{C}_{0}, k=1,2, \ldots$

Then there exists a HPD linear functional $\mathfrak{G}$ on $\mathcal{L} \cdot \mathcal{L}^{c}$ so that

$$
\langle f, g\rangle_{\mathfrak{G}}=\mathfrak{G}\left\{f g^{c}\right\}
$$

defines an inner product on $\mathcal{L}$ for which the $\chi_{k}$ form an orthonormal system.

The above three-term recurrence relation can also be written as follows:

$$
\begin{aligned}
& x\left\{\varphi_{k-1}(x)-\frac{a_{k-1}}{Z_{k-2}^{c}\left(\alpha_{k-2}\right)} \varphi_{k-2}(x)\right\}=a_{k-1}\left(1-\frac{x}{\alpha_{k-2}}\right) \varphi_{k-2}(x) \\
& \quad+b_{k-1}\left(1-\frac{x}{\alpha_{k-1}}\right) \varphi_{k-1}(x)+c_{k-1}\left(1-\frac{x}{\alpha_{k}}\right) \varphi_{k}(x), \quad 0<k \leqslant n,
\end{aligned}
$$

where

$$
b_{k-1}=-D_{k}, \quad c_{k-1}=E_{k}^{-1}, \text { and } a_{k-1}=C_{k}=\left\{1+\frac{b_{k-1}}{Z_{k-1}^{c}\left(\alpha_{k-1}\right)}\right\} \bar{c}_{k-2} .
$$

Let $\mathbf{I}_{n}$ denote the $n$ by $n$ identity matrix, and define the matrices

$$
\begin{aligned}
\mathbf{J}_{n} & =\left(\begin{array}{ccccc}
b_{0} & c_{0} & 0 & \ldots & 0 \\
a_{1} & b_{1} & c_{1} & \ddots & \vdots \\
0 & \ddots & \ddots & \ddots & 0 \\
\vdots & \ddots & a_{n-2} & b_{n-2} & c_{n-2} \\
0 & \ldots & 0 & a_{n-1} & b_{n-1}
\end{array}\right), \mathbf{D}_{n}=\operatorname{diag}\left(\alpha_{0}^{-1}, \alpha_{1}^{-1}, \ldots, \alpha_{n-1}^{-1}\right) \\
\mathbf{S}_{n} & =\left(\begin{array}{ccccc}
0 & \ldots & \ldots & \ldots & 0 \\
\frac{a_{1}}{Z_{0}^{c}\left(\alpha_{0}\right)} & \ddots & & & \vdots \\
0 & \ddots & \ddots & & \vdots \\
\vdots & \ddots & \ddots & \ddots & \vdots \\
0 & \ldots & 0 & \frac{a_{n-1}}{Z_{n-2}^{c}\left(\alpha_{n-2}\right)} & 0
\end{array}\right), \text { and } \mathbf{B}_{n}=\mathbf{J}_{n} \mathbf{D}_{n}+\mathbf{I}_{n}-\mathbf{S}_{n}
\end{aligned}
$$

and vectors $\underline{\varphi}_{n}(x)=\left(\varphi_{0}(x) \varphi_{1}(x) \ldots \varphi_{n-1}(x)\right)^{T}$ and $\underline{e}_{n}=\left(\begin{array}{lll}0 \ldots & \ldots & 1\end{array}\right)^{T} \in$ $\mathbb{C}^{n}$. Assuming the nORFs $\varphi_{k} \in{ }^{d} \mathcal{L}_{k}$ for $k=0, \ldots, n-1$, and $\varphi_{n} \in{ }^{e} \mathcal{L}_{n}$, we obtain that

$$
\mathbf{J}_{n} \underline{\varphi}_{n}(x)=x \mathbf{B}_{n} \underline{\varphi}_{n}(x)-c_{n-1}\left(1-\frac{x}{\alpha_{n}}\right) \varphi_{n}(x) \underline{e}_{n} .
$$

The following theorem has then be proved in [32, Sec. 4]. 
Theorem 3 Suppose the $n O R F s \varphi_{k} \in{ }^{d} \mathcal{L}_{k}$ for $k=0, \ldots, n-1$. Then the zeros $x_{n, j}, j=1, \ldots, n$, of a $n O R F \varphi_{n}(x) \in{ }^{e} \mathcal{L}_{n}$ are eigenvalues of the generalized eigenvalue problem (GEP)

$$
\mathbf{J}_{n} \underline{v}_{n, j}=x_{n, j} \mathbf{B}_{n} \underline{v}_{n, j},
$$

with

$$
\underline{v}_{n, j}=\eta_{n}\left\{\sum_{k=0}^{n-1}\left|\varphi_{k}\left(x_{n, j}\right)\right|^{2}\right\}^{-1 / 2} \underline{\varphi}_{n}\left(x_{n, j}\right), \quad \eta_{n} \in \mathbb{T}
$$

the corresponding normalized eigenvector.

In the remainder of this paper we will assume that the following conditions hold:

(C1) There exists a sequence $\left\{\varphi_{k}\right\}_{k=0}^{n}$, with $n>0$ and $\varphi_{k} \in{ }^{d} \mathcal{L}_{k}$ for $k=0, \ldots, n$, so that $\varphi_{k} \perp_{\mathfrak{F}} \mathcal{L}_{k-1}$ for $k=0, \ldots, n$.

(C2) There exists a function $\varphi_{n}^{\left[\bar{\alpha}_{n-1}\right]} \in \mathcal{L}_{n}^{\left[\bar{\alpha}_{n-1}\right]} \backslash \mathcal{L}_{n-1}$ (not necessarily in ${ }^{e} \mathcal{L}_{n}^{\left[\bar{\alpha}_{n-1}\right]}$ ) so that $\varphi_{n}^{\left[\bar{\alpha}_{n-1}\right]} \perp_{\mathfrak{F}} \mathcal{L}_{n-1}$.

Finally, following a similar reasoning as in [12] for the polynomial case, the zeros $x_{n, j}^{[f]}$ of an arbitrary rational function $f_{n} \in \mathcal{L}_{n} \backslash \mathcal{L}_{n-1}$, with

$$
f_{n}(x)=\sum_{k=0}^{n} \gamma_{n, k}^{[f]} \varphi_{k}(x), \quad \gamma_{n, k}^{[f]}=\left\langle f_{n}, \varphi_{k}\right\rangle_{\mathfrak{F}}
$$

can be computed from the GEP

$$
\left(\mathbf{J}_{n}-\frac{c_{n-1}}{\gamma_{n, n}^{[f]}} \underline{e}_{n} \underline{\gamma}_{f_{n}}^{T}\right) \underline{v}_{n, j}^{[f]}=x_{n, j}^{[f]}\left(\mathbf{B}_{n}-\frac{c_{n-1}}{\alpha_{n} \gamma_{n, n}^{[f]}} \underline{e}_{n} \underline{\gamma}_{f_{n}}^{T}\right) \underline{v}_{n, j}^{[f]},
$$

where

$$
\underline{\gamma}_{f_{n}}=\left(\gamma_{n, 0}^{[f]} \gamma_{n, 1}^{[f]} \ldots \gamma_{n, n-1}^{[f]}\right)^{T}
$$

and

$$
\underline{v}_{n, j}^{[f]}=\eta_{n}\left\{\sum_{k=0}^{n-1}\left|\varphi_{k}\left(x_{n, j}^{[f]}\right)\right|^{2}\right\}^{-1 / 2} \underline{\varphi}_{n}\left(x_{n, j}^{[f]}\right), \quad \eta_{n} \in \mathbb{T},
$$

is the corresponding normalized eigenvector. Note that this GEP directly follows from (7) together with

$$
\varphi_{n}(x)=\frac{1}{\gamma_{n, n}^{[f]}}\left(f_{n}(x)-\sum_{k=0}^{n-1} \gamma_{n, k}^{[f]} \varphi_{k}(x)\right), \quad \gamma_{n, n}^{[f]} \neq 0 .
$$




\section{Quasi-orthogonal rational functions}

In general, a rational function $Q_{n} \in \mathcal{L}_{n} \backslash \mathcal{L}_{n-1}$ is called a quasi-orthogonal rational function (qORF) whenever $Q_{n} \perp_{\mathfrak{F}} \mathcal{K} \subseteq \mathcal{L}_{n-1}$. The zeros of such a qORF depend on a set of parameters, which can be chosen freely, where the number of parameters depends on the subspace $\mathcal{K}$. In this paper we will consider a special kind of qORFs, namely a family of qORFs for which the zeros depend on only one parameter $\tau \in \overline{\mathbb{C}}$. More specific, we will use the following definition for qORFs.

Definition 4 We call a rational function $Q_{n} \in \mathcal{L}_{n} \backslash \mathcal{L}_{n-1}, n>0$, a qORF iff $Q_{n} \perp_{\mathfrak{F}} \mathcal{L}_{n-1}\left(\bar{\alpha}_{n}\right)$.

In theorem 5 below we will show that the zeros of this $Q_{n}$ indeed will depend on one parameter $\tau_{n} \in \overline{\mathbb{C}}$. As we will see later on in Section 5 , the main importance of $Q_{n}$ is that it is always possible to choose the parameter $\tau_{n} \in \overline{\mathbb{C}}$ in such a way that all the zeros are real and simple, and hence, can be used as nodes in an $n$-point rational quadrature rule. Moreover, if $\alpha_{n} \notin \overline{\mathbb{R}}$, this $n$-point rational quadrature rule will have the maximal possible domain of validity in the space of rational functions with poles among $\mathcal{A}$. The following theorem now gives a characterization for qORFs in terms of nORFs.

Theorem 5 A rational function $Q_{n} \in \mathcal{L}_{n} \backslash \mathcal{L}_{n-1}$ is a qORF iff there exist constants $A_{n}^{[Q]}, B_{n}^{[Q]} \in \mathbb{C}$ so that

$$
Q_{n}(x)=A_{n}^{[Q]} \varphi_{n}(x)+B_{n}^{[Q]} \frac{Z_{n}(x)}{Z_{n-1}^{c}(x)} \varphi_{n-1}(x),
$$

with

$$
A_{n}^{[Q]} \neq \frac{B_{n}^{[Q]}}{Z_{n}\left(\bar{\alpha}_{n-1}\right)}\left\langle Z_{n} \varphi_{n-1}, \varphi_{n}\right\rangle_{\mathfrak{F}}=-B_{n}^{[Q]}\left[\frac{Z_{n}(x) \varphi_{n-1}(x)}{Z_{n-1}^{c}(x) \varphi_{n}(x)}\right]_{x=\alpha_{n}} .
$$

Proof. Since $Q_{n} \perp_{\mathfrak{F}} \mathcal{L}_{n-1}\left(\bar{\alpha}_{n}\right)$, it follows that $\frac{Z_{n-1}^{c}}{Z_{n}} Q_{n} \in \mathcal{L}_{n}^{\left[\bar{\alpha}_{n-1}\right]}$ and $\frac{Z_{n-1}^{c}}{Z_{n}} Q_{n} \perp_{\mathfrak{F}}$ $\mathcal{L}_{n-2}$. Consider now the nORF $\varphi_{n}^{\left[\bar{\alpha}_{n-1}\right]} \in \mathcal{L}_{n}^{\left[\bar{\alpha}_{n-1}\right]} \backslash \mathcal{L}_{n-1}$. Then there exist constants $\tilde{A}_{n}, \tilde{B}_{n} \in \mathbb{C}$ so that

$$
\frac{Z_{n-1}^{c}(x)}{Z_{n}(x)} Q_{n}(x)=\tilde{A}_{n} \varphi_{n}^{\left[\bar{\alpha}_{n-1}\right]}(x)+\tilde{B}_{n} \varphi_{n-1}(x),
$$

and hence

$$
Q_{n}(x)=\tilde{A}_{n} \frac{Z_{n}(x)}{Z_{n-1}^{c}(x)} \varphi_{n}^{\left[\bar{\alpha}_{n-1}\right]}(x)+\tilde{B}_{n} \frac{Z_{n}(x)}{Z_{n-1}^{c}(x)} \varphi_{n-1}(x) .
$$

Assuming $Q_{n} \in \mathcal{L}_{n} \backslash \mathcal{L}_{n-1}$, it follows that there exists a constant $\gamma_{n, n}^{[Q]}:=$ $\left\langle Q_{n}, \varphi_{n}\right\rangle_{\mathfrak{F}} \neq 0$ and a function $f_{n-1} \in \mathcal{L}_{n-1}$ so that $Q_{n}(x)=\gamma_{n, n}^{[Q]} \varphi_{n}(x)+$ 
$f_{n-1}(x)$. Moreover, since $\varphi_{n} \perp_{\mathfrak{F}} \mathcal{L}_{n-1} \supset \mathcal{L}_{n-1}\left(\bar{\alpha}_{n}\right)$, it follows that $f_{n-1} \perp_{\mathfrak{F}}$ $\mathcal{L}_{n-1}\left(\bar{\alpha}_{n}\right)$; hence, $f_{n-1}$ is of the form

$$
f_{n-1}(x)=\hat{A}_{n} \frac{Z_{n}(x)}{Z_{n-1}^{c}(x)} \varphi_{n}^{\left[\bar{\alpha}_{n-1}\right]}(x)+\hat{B}_{n} \frac{Z_{n}(x)}{Z_{n-1}^{c}(x)} \varphi_{n-1}(x), \quad \hat{A}_{n}, \hat{B}_{n} \in \mathbb{C},
$$

where $\hat{A}_{n} \neq \tilde{A}_{n}$. Indeed, for $\hat{A}_{n}=\tilde{A}_{n}$ we would get that

$$
\gamma_{n, n}^{[Q]} \varphi_{n}(x)=\left(\tilde{B}_{n}-\hat{B}_{n}\right) \frac{Z_{n}(x)}{Z_{n-1}^{c}(x)} \varphi_{n-1}(x),
$$

which is impossible due to the fact that $\varphi_{n} \in{ }^{d} \mathcal{L}_{n}$. Thus,

$$
\tilde{A}_{n} \frac{Z_{n}(x)}{Z_{n-1}^{c}(x)} \varphi_{n}^{\left[\bar{\alpha}_{n-1}\right]}(x)=A_{n}^{[Q]} \varphi_{n}(x)+\left(B_{n}^{[Q]}-\tilde{B}_{n}\right) \frac{Z_{n}(x)}{Z_{n-1}^{c}(x)} \varphi_{n-1}(x),
$$

with

$$
A_{n}^{[Q]}=\frac{\tilde{A}_{n} \cdot \gamma_{n, n}^{[Q]}}{\tilde{A}_{n}-\hat{A}_{n}} \in \mathbb{C} \quad \text { and } \quad B_{n}^{[Q]}=\tilde{B}_{n}+\frac{\tilde{A}_{n} \cdot\left(\hat{B}_{n}-\tilde{B}_{n}\right)}{\tilde{A}_{n}-\hat{A}_{n}} \in \mathbb{C},
$$

which leads to the equality in (11). Finally, for $Q_{n} \in \mathcal{L}_{n} \backslash \mathcal{L}_{n-1}$ it holds that $\gamma_{n, n}^{[Q]}=A_{n}^{[Q]}+B_{n}^{[Q]}\left\langle\frac{Z_{n}}{Z_{n-1}^{c}} \varphi_{n-1}, \varphi_{n}\right\rangle_{\mathfrak{F}}=A_{n}^{[Q]}+\frac{B_{n}^{[Q]}}{Z_{n-1}^{c}\left(\alpha_{n}\right)}\left\langle Z_{n} \varphi_{n-1}, \varphi_{n}\right\rangle_{\mathfrak{F}} \neq 0$.

This ends the proof.

From the previous theorem it clearly follows that the zeros of a qORF $Q_{n} \in \mathcal{L}_{n} \backslash \mathcal{L}_{n-1}$ depend on one single parameter

$$
\tau_{n}=\frac{B_{n}^{[Q]}}{A_{n}^{[Q]}}=: \tau_{n}^{[Q]} \in \overline{\mathbb{C}}
$$

The following two theorems will be important in order to prove the existence of values $\tau_{n}^{[Q]} \in \overline{\mathbb{C}}$ so that the zeros of $Q_{n}$ are all simple and real, and to provide a characterization for those values $\tau_{n}^{[Q]}$.

Theorem 6 A qORF $Q_{n} \in{ }^{e} \mathcal{L}_{n}$ (i.e., $\tau_{n}^{[Q]}\left[\frac{Z_{n}(x) \varphi_{n-1}(x)}{Z_{n-1}^{c}(x) \varphi_{n}(x)}\right]_{x=\alpha_{n-1}} \neq-1$ ) satisfies a relation of the form

$$
Q_{n}(x)=E_{n}^{[Q]} Z_{n}(x)\left\{\left[1+\frac{D_{n}^{[Q]}}{Z_{n-1}(x)}\right] \varphi_{n-1}(x)-\frac{C_{n}^{[Q]}}{Z_{n-2}^{c}(x)} \varphi_{n-2}(x)\right\}
$$

with $E_{n}^{[Q]} \neq 0$. The coefficients $E_{n}^{[Q]}, D_{n}^{[Q]}$ and $C_{n}^{[Q]}$ are given by

$$
\begin{aligned}
E_{n}^{[Q]} & =A_{n}^{[Q]} E_{n}+B_{n}^{[Q]} / Z_{n-1}^{c}\left(\alpha_{n-1}\right) \\
D_{n}^{[Q]} & =\frac{A_{n}^{[Q]} E_{n} D_{n}+B_{n}^{[Q]}}{A_{n}^{[Q]} E_{n}+B_{n}^{[Q]} / Z_{n-1}^{c}\left(\alpha_{n-1}\right)} \\
C_{n}^{[Q]} & =\frac{A_{n}^{[Q]} E_{n} C_{n}}{A_{n}^{[Q]} E_{n}+B_{n}^{[Q]} / Z_{n-1}^{c}\left(\alpha_{n-1}\right)},
\end{aligned}
$$


where the coefficients $C_{n}^{[Q]}$ and $D_{n}^{[Q]}$ are related by

$$
C_{n}^{[Q]}=\frac{1-D_{n}^{[Q]} / Z_{n-1}^{c}\left(\alpha_{n-1}\right)}{\bar{E}_{n-1}} .
$$

Further, $C_{n}^{[Q]} \neq 0$ iff $Q_{n}$ is not degenerate (i.e., $A_{n}^{[Q]} \neq 0$ ); hence, iff $Q_{n} \in{ }^{d} \mathcal{L}_{n}$.

Proof. From (11) together with (5) we deduce that

$$
\begin{aligned}
Q_{n}(x)=A_{n}^{[Q]} & E_{n} Z_{n}(x)\left\{\left[1+\frac{D_{n}}{Z_{n-1}(x)}\right] \varphi_{n-1}(x)-\frac{C_{n}}{Z_{n-2}^{c}(x)} \varphi_{n-2}(x)\right\} \\
& +B_{n}^{[Q]} Z_{n}(x)\left[\frac{1}{Z_{n-1}^{c}\left(\alpha_{n-1}\right)}+\frac{1}{Z_{n-1}(x)}\right] \varphi_{n-1}(x) \\
& =Z_{n}(x)\left\{\left[E_{n}^{[Q]}+\frac{\tilde{D}_{n}}{Z_{n-1}(x)}\right] \varphi_{n-1}(x)-\frac{\tilde{C}_{n}}{Z_{n-2}^{c}(x)} \varphi_{n-2}(x)\right\},
\end{aligned}
$$

where $E_{n}^{[Q]}$ is given by (13), and

$$
\tilde{D}_{n}=A_{n}^{[Q]} E_{n} D_{n}+B_{n}^{[Q]} \text { and } \quad \tilde{C}_{n}=A_{n}^{[Q]} E_{n} C_{n} .
$$

Further, for $Q_{n}=\frac{q_{n}}{\pi_{n}} \in{ }^{e} \mathcal{L}_{n}$ we find with $\varphi_{k}=\frac{p_{k}}{\pi_{k}}$ that

$$
q_{n}\left(\alpha_{n-1}\right)=E_{n}^{[Q]} \alpha_{n-1} p_{n-1}\left(\alpha_{n-1}\right) \neq 0,
$$

so that $E_{n}^{[Q]} \neq 0$.

Next, we have that

$$
\begin{aligned}
C_{n}^{[Q]} & =\frac{A_{n}^{[Q]} E_{n} C_{n}}{A_{n}^{[Q]} E_{n}+B_{n}^{[Q]} / Z_{n-1}^{c}\left(\alpha_{n-1}\right)}=\frac{A_{n}^{[Q]} E_{n}\left[1-D_{n} / Z_{n-1}^{c}\left(\alpha_{n-1}\right)\right]}{A_{n}^{[Q]} E_{n}+B_{n}^{[Q]} / Z_{n-1}^{c}\left(\alpha_{n-1}\right)} \bar{E}_{n-1}^{-1} \\
& =\left[1-\frac{\left(A_{n}^{[Q]} E_{n} D_{n}+B_{n}^{[Q]}\right) / Z_{n-1}^{c}\left(\alpha_{n-1}\right)}{A_{n}^{[Q]} E_{n}+B_{n}^{[Q]} / Z_{n-1}^{c}\left(\alpha_{n-1}\right)}\right] \bar{E}_{n-1}^{-1} \\
& =\frac{1-D_{n}^{[Q]} / Z_{n-1}^{c}\left(\alpha_{n-1}\right)}{\bar{E}_{n-1}} .
\end{aligned}
$$

Finally, we deduce from $(15)$ that $C_{n}^{[Q]} \neq 0$ iff $A_{n}^{[Q]} \neq 0$. Since

$$
q_{n}\left(\bar{\alpha}_{n-1}\right)=A_{n}^{[Q]} p_{n}\left(\bar{\alpha}_{n-1}\right) \neq 0 \quad \text { iff } \quad A_{n}^{[Q]} \neq 0,
$$

this means that $C_{n}^{[Q]} \neq 0$ iff $Q_{n} \in{ }^{d} \mathcal{L}_{n}$.

As a consequence of Theorems 6 and 2, we now can prove the following. 
Theorem 7 Suppose the $q$ ORF $Q_{n}=\frac{q_{n}}{\pi_{n}} \in{ }^{d} \mathcal{L}_{n}$, and let $\alpha \in \overline{\mathbb{C}}_{0}$ be chosen in such a way that $q_{n}(\alpha) \neq 0$ and $\mathfrak{T}\{\alpha\}=k \cdot v_{n}$ for some $k \in \mathbb{R}_{0}^{+}$, where the constant $v_{n}$ is defined by

$$
v_{n}= \begin{cases}\Im\left\{D_{n}^{[Q]}\right\}-\frac{\mathfrak{T}\left\{\alpha_{n-2}\right\}}{\left|E_{n-1}\right|^{2}}, & \alpha_{n-1} \in \overline{\mathbb{R}}_{0} \\ s_{n}\left\{1-\left[X_{n}^{2}+\left(Y_{n}-1\right)^{2}\right] \frac{\Delta_{n-1}}{\left|E_{n-1}\right|^{2}}\right\} & \alpha_{n-1} \notin \overline{\mathbb{R}}\end{cases}
$$

with

$$
X_{n}=2 \Re\left\{D_{n}^{[Q]}\right\} \cdot \mathfrak{T}\left\{\alpha_{n-1}\right\}, \quad Y_{n}=2 \Im\left\{D_{n}^{[Q]}\right\} \cdot \mathfrak{T}\left\{\alpha_{n-1}\right\},
$$

and $s_{n}=\operatorname{sign}\left(\Im\left\{\alpha_{n-1}\right\}\right)$. Then $\frac{Z_{\alpha}}{Z_{n}} Q_{n} \in{ }^{d} \mathcal{L}_{n}^{[\alpha]}$ is orthogonal on $\mathcal{L}_{n-1}$ with respect to an inner product defined by an HPD linear functional $\mathfrak{G}$ :

$$
\langle f, g\rangle_{\mathfrak{G}}=\mathfrak{G}\left\{f g^{c}\right\},
$$

not necessarily the same as the HPD linear functional $\mathfrak{F}$, but for which $\left\{\varphi_{k}\right\}_{k=0}^{n-1}$ are nORFs too:

$$
\left\langle\varphi_{k}, \varphi_{j}\right\rangle_{\mathfrak{G}}=\delta_{k, j}, \quad k, j=0, \ldots, n-1 .
$$

Proof. Let $\varphi_{n}^{[\alpha]}$ be given by

$$
\begin{aligned}
& \varphi_{n}^{[\alpha]}(x)=\frac{E_{n}^{[\alpha]}}{E_{n}^{[Q]}} \cdot \frac{Z_{\alpha}(x)}{Z_{n}(x)} Q_{n}(x) \\
= & E_{n}^{[\alpha]} Z_{\alpha}(x)\left\{\left[1+\frac{D_{n}^{[Q]}}{Z_{n-1}(x)}\right] \varphi_{n-1}(x)-\frac{C_{n}^{[Q]}}{Z_{n-2}^{c}(x)} \varphi_{n-2}(x)\right\}, \quad E_{n}^{[\alpha]} \in \mathbb{C}_{0} .
\end{aligned}
$$

Then it holds that $\varphi_{n}^{[\alpha]} \in \mathcal{L}_{n}^{[\alpha]} \backslash \mathcal{L}_{n-1}$. Hence, together with (16) and the fact that $C_{n}^{[Q]} \neq 0$ it follows that assumptions (A1)-(A4) and (A6) in Theorem 2 are satisfied. Next, if $v_{n}=0$, it follows that assumption (A5) in Theorem 2 is satisfied too for every $E_{n}^{[\alpha]} \in \mathbb{C}_{0}$. If, on the other hand, $v_{n} \neq 0$, it follows that assumption (A5) in Theorem 2 is satisfied for

$$
\left|E_{n}^{[\alpha]}\right|^{2}=\left\{\begin{array}{lr}
k, & \alpha_{n-1} \in \overline{\mathbb{R}}_{0} \\
4 k\left|\mathfrak{T}\left\{\alpha_{n-1}\right\}\right|, & \alpha_{n-1} \notin \overline{\mathbb{R}}
\end{array}\right.
$$

with

$$
\Delta_{n}^{[\alpha]}= \begin{cases}k, & \alpha_{n-1} \in \overline{\mathbb{R}}_{0} \\ \left\{X_{n}^{2}+\left(Y_{n}-1\right)^{2}\right\} \frac{4 k \Delta_{n-1}\left|\mathfrak{T}\left\{\alpha_{n-1}\right\}\right|}{\left|E_{n-1}\right|^{2}}, & \alpha_{n-1} \notin \overline{\mathbb{R}} .\end{cases}
$$

From the definition of $v_{n}$ in the previous theorem it is clear that $v_{n} \in \mathbb{R}$, so that the condition on $\mathfrak{T}\{\alpha\}$ is equivalent with

$$
\alpha \in\left\{\begin{array}{lll}
\mathbb{U}:=\{z \in \mathbb{C}: \Im\{z\}>0\} & \text { if } & v_{n}>0 \\
\mathbb{R}_{0} & \text { if } & v_{n}=0 \\
\mathbb{L}:=\{z \in \mathbb{C}: \Im\{z\}<0\} & \text { if } & v_{n}<0 .
\end{array}\right.
$$


Due to this, and because a qORF $Q_{n} \in \mathcal{L}_{n}$ has a finite number of zeros, the auxiliary pole $\alpha$ always exists and is not unique. For every $\alpha$ that satisfies the conditions in the theorem, the factor $Z_{\alpha} / Z_{n}$ merely replaces the last pole of $Q_{n}$ with a pole in $\alpha$. Hence, $Q_{n}$ and $\frac{Z_{\alpha}}{Z_{n}} Q_{n}$ have the same zeros, while the latter is (up to a non-zero multiplicative factor) a nORF in $\mathcal{L}_{n}^{[\alpha]} \backslash \mathcal{L}_{n-1}$ if $Q_{n} \in{ }^{d} \mathcal{L}_{n}$. Hence, the previous theorem shows that the zeros of a qORF $Q_{n} \in{ }^{d} \mathcal{L}_{n}$ are in fact the zeros of a certain nORF. Under certain conditions, the opposite holds as well, as will be proved in the next theorem.

Theorem 8 Let $\varphi_{n}^{[\alpha]} \in{ }^{d} \mathcal{L}_{n}^{[\alpha]}$ represent a nORF with respect to an HPD linear functional $\mathfrak{G}$. Assume the polynomial in the numerator of $\varphi_{n}^{[\alpha]}$ has no zero in $\alpha_{n}$, and suppose the rational functions $\varphi_{n-2}$ and $\varphi_{n-1}$ are $n$ ORFs with respect to the HPD linear functional $\mathfrak{G}$ too, such that there exist constants $E_{n}^{[\alpha]} \in \mathbb{C}_{0}$ and $D_{n}^{[\alpha]} \in \mathbb{C}$ so that

$$
\varphi_{n}^{[\alpha]}(x)=E_{n}^{[\alpha]} Z_{n}(x)\left\{\left[1+\frac{D_{n}^{[\alpha]}}{Z_{n-1}(x)}\right] \varphi_{n-1}(x)-\frac{C_{n}^{[\alpha]}}{Z_{n-2}^{c}(x)} \varphi_{n-2}(x)\right\},
$$

with

$$
C_{n}^{[\alpha]}=\frac{1-D_{n}^{[\alpha]} / Z_{n-1}^{c}\left(\alpha_{n-1}\right)}{\bar{E}_{n-1}} \in \mathbb{C}_{0} .
$$

Then, $\frac{Z_{n}}{Z_{\alpha}} \varphi_{n}^{[\alpha]} \in{ }^{d} \mathcal{L}_{n}$ is a qORF with coefficients

$$
A_{n}^{[Q]}=\frac{E_{n}^{[\alpha]} C_{n}^{[\alpha]}}{E_{n} C_{n}} \quad \text { and } \quad B_{n}^{[Q]}=\frac{E_{n}^{[\alpha]}\left(D_{n}^{[\alpha]}-D_{n}\right)}{\bar{E}_{n-1} C_{n}} .
$$

Proof. The statement directly follows from (12) by considering the system of equations

$$
\left\{\begin{array}{l}
E_{n}^{[Q]}=E_{n}^{[\alpha]} \\
D_{n}^{[Q]}=D_{n}^{[\alpha]}
\end{array}\right.
$$

and solving this system of equations for $A_{n}^{[Q]}$ and $B_{n}^{[Q]}$ by means of (13)-(15).

Finally, let $\gamma_{n, k}^{[Z \varphi]}$ denote the $k$-th projection coefficient of $Z_{n} \varphi_{n-1}$ onto the basis $\left\{\varphi_{0}, \ldots, \varphi_{n}\right\}$, i.e.,

$$
\gamma_{n, k}^{[Z \varphi]}:=\left\langle Z_{n} \varphi_{n-1}, \varphi_{k}\right\rangle_{\mathfrak{F}}, \quad k=0, \ldots, n .
$$

One way to compute the zeros of a qORF $Q_{n} \in \mathcal{L}_{n} \backslash \mathcal{L}_{n-1}$, is by means of the GEP (10), with

$$
\gamma_{n, k}^{[Q]}:=\left\langle Q_{n}, \varphi_{k}\right\rangle_{\mathfrak{F}}=A_{n}^{[Q]} \delta_{n, k}+B_{n}^{[Q]} \delta_{n-1, k}+\frac{B_{n}^{[Q]}}{Z_{n-1}^{c}\left(\alpha_{n}\right)} \gamma_{n, k}^{[Z \varphi]}, \quad k=0, \ldots, n .
$$


Whenever $Q_{n} \in{ }^{e} \mathcal{L}_{n}$, we deduce from Theorem 6 that

$$
\begin{array}{r}
x\left\{\varphi_{n-1}(x)-\frac{a_{n-1}^{[Q]}}{Z_{n-2}^{c}\left(\alpha_{n-2}\right)} \varphi_{n-2}(x)\right\}=a_{n-1}^{[Q]}\left(1-\frac{x}{\alpha_{n-2}}\right) \varphi_{n-2}(x) \\
+b_{n-1}^{[Q]}\left(1-\frac{x}{\alpha_{n-1}}\right) \varphi_{n-1}(x)+c_{n-1}^{[Q]}\left(1-\frac{x}{\alpha_{n}}\right) Q_{n}(x),
\end{array}
$$

with

$b_{n-1}^{[Q]}=\frac{A_{n}^{[Q]} b_{n-1}-B_{n}^{[Q]} c_{n-1}}{A_{n}^{[Q]}+B_{n}^{[Q]} c_{n-1} / Z_{n-1}^{c}\left(\alpha_{n-1}\right)}, \quad c_{n-1}^{[Q]}=\frac{c_{n-1}}{A_{n}^{[Q]}+B_{n}^{[Q]} c_{n-1} / Z_{n-1}^{c}\left(\alpha_{n-1}\right)}$,

and

$$
a_{n-1}^{[Q]}=\frac{A_{n}^{[Q]} a_{n-1}}{A_{n}^{[Q]}+B_{n}^{[Q]} c_{n-1} / Z_{n-1}^{c}\left(\alpha_{n-1}\right)},
$$

where $a_{n-1}, b_{n-1}$ and $c_{n-1}$ are defined as before in (8). So, let us now define

$$
\mathbf{J}_{n}^{[Q]}=\left(\begin{array}{cc}
\mathbf{J}_{n-1} & c_{n-2} \cdot \underline{e}_{n-1} \\
a_{n-1}^{[Q]} \cdot \underline{e}_{n-1}^{T} & b_{n-1}^{[Q]}
\end{array}\right), \quad \mathbf{S}_{n}^{[Q]}=\left(\begin{array}{cc}
\mathbf{S}_{n-1} & \underline{0}_{n-1} \\
\frac{a_{n-1}^{[Q]}}{Z_{n-2}^{c}\left(\alpha_{n-2}\right)} \cdot \underline{e}_{n-1}^{T} & 0
\end{array}\right),
$$

where $\underline{0}_{n-1}$ denotes the zero-vector in $\mathbb{C}^{n-1}$, and $\mathbf{B}_{n}^{[Q]}=\mathbf{J}_{n}^{[Q]} \mathbf{D}_{n}+\mathbf{I}_{n}-\mathbf{S}_{n}^{[Q]}$. Then the zeros $x_{n, j}^{[Q]}, j=1, \ldots, n$, of the $\mathrm{qORF} Q_{n}(x) \in{ }^{e} \mathcal{L}_{n}$ are eigenvalues of the GEP

$$
\mathbf{J}_{n}^{[Q]} \underline{v}_{n, j}^{[Q]}=x_{n, j}^{[Q]} \mathbf{B}_{n}^{[Q]} \underline{v}_{n, j}^{[Q]},
$$

with

$$
\underline{v}_{n, j}^{[Q]}=\eta_{n}\left\{\sum_{k=0}^{n-1}\left|\varphi_{k}\left(x_{n, j}^{[Q]}\right)\right|^{2}\right\}^{-1 / 2} \underline{\varphi}_{n}\left(x_{n, j}^{[Q]}\right), \quad \eta_{n} \in \mathbb{T},
$$

the corresponding normalized eigenvector.

Remark 9 If $Q_{n} \in{ }^{e} \mathcal{L}_{n} \backslash{ }^{d} \mathcal{L}_{n}$, it follows from Theorem 6 that $A_{n}^{[Q]}=0$. From (11) we then deduce that, besides the zero in $\bar{\alpha}_{n-1}$, the remaining zeros of $Q_{n}$ are zeros of $\varphi_{n-1}$. So, in this case the GEP (9) can be used instead to compute these remaining zeros.

Remark 10 For the construction of a rational Gaussian quadrature rule, we are only interested in qORFs $Q_{n} \in{ }^{r} \mathcal{L}_{n}$ (see Section 5), i.e., $\tau_{n}^{[Q]} \in \mathbb{C}$ and

$$
\tau_{n}^{[Q]}\left[\frac{Z_{n}(x) \varphi_{n-1}(x)}{Z_{n-1}^{c}(x) \varphi_{n}(x)}\right]_{x=\alpha_{k}} \neq-1, \quad k=0, \ldots, n .
$$




\section{Para-orthogonal rational functions}

Whenever the HPD linear functional $\mathfrak{F}$ is defined by a positive bounded Borel measure $\mu$ with infinite $\operatorname{support} \operatorname{supp}(\mu) \subseteq[a, b]$, where $-\infty \leqslant a<b \leqslant \infty$ and $[a, b] \subsetneq \overline{\mathbb{R}}$, and the poles are outside $[a, b]$, one mostly prefers to work with so-called para-orthogonal rational functions (pORFs) to construct a rational quadrature rule with the maximal possible domain of validity in the space of rational functions with poles among $\mathcal{A}$. Again, the zeros of a pORF $P_{n} \in$ $\mathcal{L}_{n} \backslash \mathcal{L}_{n-1}$ depend on one parameter $\tau \in \overline{\mathbb{C}}$, but the main advantage of pORFs over qORFs is that this parameter is easily characterized to ensure all the zeros of $P_{n}$ are simple and inside $[a, b]$. In the most general way, a pORF can be defined as follows.

Definition 11 We call a rational function $P_{n} \in \mathcal{L}_{n} \backslash \mathcal{L}_{n-1}, n>0$, a pORF iff there exist constants $A_{n}^{[P]}, B_{n}^{[P]} \in \mathbb{C}$, so that

$$
P_{n}(x)=A_{n}^{[P]} \varphi_{n}(x)+B_{n}^{[P]} \varphi_{n}^{*}(x),
$$

with

$$
A_{n}^{[P]} \neq-B_{n}^{[P]}\left\langle\varphi_{n}, \varphi_{n}^{*}\right\rangle_{\mathfrak{F}}=-B_{n}^{[P]}\left[\frac{\varphi_{n}^{*}(x)}{\varphi_{n}(x)}\right]_{x=\alpha_{n}} .
$$

From this definition it clearly follows that the zeros of a pORF $P_{n} \in$ $\mathcal{L}_{n} \backslash \mathcal{L}_{n-1}$ depend on one single parameter

$$
\tau_{n}=\frac{B_{n}^{[P]}}{A_{n}^{[P]}}=: \tau_{n}^{[P]} \in \overline{\mathbb{C}}
$$

In Theorem 13 we will prove a connection between pORFs and qORFs, but first we need the following lemma.

Lemma 12 The zeros of a $n O R F \varphi_{n} \in{ }^{d} \mathcal{L}_{n}$ are all real iff $\alpha_{n} \in \overline{\mathbb{R}}_{0}$.

Proof. For $\alpha_{n} \in \overline{\mathbb{R}}_{0}$ it has already been stated in [37, Lem. 3.1] $]^{2}$ that the zeros of $\varphi_{n}$ are all real, so it remains to prove that they are not all real whenever $\alpha_{n} \notin \overline{\mathbb{R}}$. Suppose that $\alpha_{n} \notin \overline{\mathbb{R}}$, and assume that the zeros of $\varphi_{n}$ are all real. It then holds that there exists a constant $\kappa_{n} \in \mathbb{T}$ so that

$$
\varphi_{n}^{*}(x)=\kappa_{n} \varphi_{n}(x),
$$

and hence, $\varphi_{n}^{*} \perp_{\mathfrak{F}} \mathcal{L}_{n-1}$. From

$$
0=\left\langle\varphi_{n}^{*}, \mathcal{L}_{n-1}\right\rangle_{\mathfrak{F}}=\left\langle\frac{Z_{n}}{Z_{n}^{c}} \varphi_{n}^{c}, \mathcal{L}_{n-1}^{c}\right\rangle_{\mathfrak{F}}=\overline{\left\langle\mathcal{L}_{n-1}, \frac{Z_{n}^{c}}{Z_{n}} \varphi_{n}\right\rangle_{\mathfrak{F}}},
$$

\footnotetext{
2 The statement in [37, Lem. 3.1] has been proved in the case of complex poles in the lower half-plane in [6, Cor. 3.2.2], but this proof remains valid for arbitrary complex poles.
} 
we deduce that $\phi_{n}^{\left[\bar{\alpha}_{n}\right]}:=\frac{Z_{n}^{c}}{Z_{n}} \varphi_{n} \in{ }^{d} \mathcal{L}_{n}^{\left[\bar{\alpha}_{n}\right]}$ and $\phi_{n}^{\left[\bar{\alpha}_{n}\right]} \perp_{\mathfrak{F}} \mathcal{L}_{n-1}$. So, let $\varphi_{n}^{\left[\bar{\alpha}_{n}\right]}(x)=$ $k_{n} \phi_{n}^{\left[\bar{\alpha}_{n}\right]}(x)$, with $k_{n} \in \mathbb{C}_{0}$ such that $\left\|\varphi_{n}^{\left[\bar{\alpha}_{n}\right]}\right\|_{\mathfrak{F}}=1$. From Theorem 1 it follows that there exist constants $E_{n}^{\left[\bar{\alpha}_{n}\right]} \in \mathbb{C}_{0}$ and $D_{n}^{\left[\bar{\alpha}_{n}\right]} \in \mathbb{C}$ so that

$$
\varphi_{n}^{\left[\bar{\alpha}_{n}\right]}(x)=E_{n}^{\left[\bar{\alpha}_{n}\right]} Z_{n}^{c}(x)\left\{\left[1+\frac{D_{n}^{\left[\bar{\alpha}_{n}\right]}}{Z_{n-1}(x)}\right] \varphi_{n-1}(x)-\frac{C_{n}^{\left[\bar{\alpha}_{n}\right]}}{Z_{n-2}^{c}(x)} \varphi_{n-2}(x)\right\}
$$

with

$$
C_{n}^{\left[\bar{\alpha}_{n}\right]}=\frac{1-D_{n}^{\left[\bar{\alpha}_{n}\right]} / Z_{n-1}^{c}\left(\alpha_{n-1}\right)}{\bar{E}_{n-1}} \in \mathbb{C}_{0}
$$

By definition we also have that

$$
\varphi_{n}^{\left[\bar{\alpha}_{n}\right]}(x)=k_{n} E_{n} Z_{n}^{c}(x)\left\{\left[1+\frac{D_{n}}{Z_{n-1}(x)}\right] \varphi_{n-1}(x)-\frac{C_{n}}{Z_{n-2}^{c}(x)} \varphi_{n-2}(x)\right\} .
$$

Taking the inner product of (24) and (25) with $\varphi_{k}$, for $k<n$, we find that $D_{n}^{\left[\bar{\alpha}_{n}\right]}=D_{n}$ and $C_{n}^{\left[\bar{\alpha}_{n}\right]}=C_{n}$. Consequently, $E_{n}^{\left[\bar{\alpha}_{n}\right]}=k_{n} E_{n}$, and hence, $\left|E_{n}^{\left[\bar{\alpha}_{n}\right]}\right|^{2}=\left|k_{n}\right|^{2}\left|E_{n}\right|^{2}$. On the other hand, from (6) it follows that $\left|E_{n}^{\left[\bar{\alpha}_{n}\right]}\right|^{2}=$ $-\left|E_{n}\right|^{2}$ due to the fact that $\mathfrak{T}\left\{\bar{\alpha}_{n}\right\}=-\mathfrak{T}\left\{\alpha_{n}\right\}$, implying that $\left|k_{n}\right|^{2}=-1$. Clearly, this is impossible.

Theorem 13 A pORF $P_{n} \in \mathcal{L}_{n} \backslash \mathcal{L}_{n-1}$ is a qORF. ${ }^{3}$ Conversely, a qORF $Q_{n} \in \mathcal{L}_{n} \backslash \mathcal{L}_{n-1}$ is a pORF iff either $\alpha_{n} \notin \overline{\mathbb{R}}$ or $B_{n}^{[Q]}=0$.

Proof. Clearly, $\varphi_{n} \perp_{\mathfrak{F}} \mathcal{L}_{n-1}\left(\bar{\alpha}_{n}\right)$, while

$$
\left\langle\varphi_{n}^{*}, \frac{Z_{n-1}}{Z_{n}^{c}} \mathcal{L}_{n-2}\right\rangle_{\mathfrak{F}}=\left\langle\frac{b_{n}}{b_{n}^{c}} \varphi_{n}^{c}, \frac{Z_{n-1}}{Z_{n}^{c}} \mathcal{L}_{n-2}\right\rangle_{\mathfrak{F}}=\left\langle\varphi_{n}^{c}, \frac{Z_{n-1}^{c}}{Z_{n}} \mathcal{L}_{n-2}^{c}\right\rangle_{\mathfrak{F}}=0,
$$

so that $\varphi_{n}^{*} \perp_{\mathfrak{F}} \mathcal{L}_{n-1}\left(\bar{\alpha}_{n}\right)$ too. Consequently, from Theorem 5 it follows that there exist constants $A_{n}^{*}$ and $B_{n}^{*}$ so that

$$
\varphi_{n}^{*}(x)=A_{n}^{*} \varphi_{n}(x)+B_{n}^{*} \frac{Z_{n}(x)}{Z_{n-1}^{c}(x)} \varphi_{n-1}(x) .
$$

So, we have proved that a pORF is a qORF, and hence, it remains to prove that a qORF is a pORF whenever either $\alpha_{n} \notin \overline{\mathbb{R}}$ or $B_{n}^{[Q]}=0$.

For $\alpha_{n} \in \overline{\mathbb{R}}_{0}$ it holds that $\varphi_{n}^{*}(x)=A_{n}^{*} \varphi_{n}(x)$, while $\frac{Z_{n}(x)}{Z_{n-1}^{c}(x)} \varphi_{n-1}(x) \neq$ $k \varphi_{n}(x)$ for every $k \in \mathbb{C}$. So, the statement clearly holds for $\alpha_{n} \in \overline{\mathbb{R}}_{0}$ with $B_{n}^{[Q]}=0$.

For $\alpha_{n} \notin \overline{\mathbb{R}}$ it follows from the previous lemma that $B_{n}^{*} \neq 0$, so that

$$
Q_{n}(x)=A_{n}^{[Q]} \varphi_{n}(x)+B_{n}^{[Q]} \frac{Z_{n}(x)}{Z_{n-1}^{c}(x)} \varphi_{n-1}(x)=A_{n}^{[P]} \varphi_{n}(x)+B_{n}^{[P]} \varphi_{n}^{*}(x),
$$

\footnotetext{
3 This statement has already been made in [37], but the proof appears not to have been written out anywhere yet.
} 
with

$$
A_{n}^{[P]}=A_{n}^{[Q]}-A_{n}^{*} \frac{B^{[Q]}}{B_{n}^{*}} \quad \text { and } \quad B^{[P]}=\frac{B^{[Q]}}{B_{n}^{*}} .
$$

For a pORF $P_{n} \in{ }^{r} \mathcal{L}_{n}$ it holds that

$$
\tau_{n}^{[P]}\left[\frac{\varphi_{n}^{*}(x)}{\varphi_{n}(x)}\right]_{x=\alpha_{k}} \neq-1, \quad k=0, \ldots, n, \quad \text { and } \quad \tau_{n}^{[P]}\left[\frac{\varphi_{n}^{*}(x)}{\varphi_{n}(x)}\right]_{x=\bar{\alpha}_{n-1}} \neq-1 .
$$

Let $\gamma_{n, k}^{\left[\varphi^{*}\right]}$ denote the $k$-th projection coefficient of $\varphi_{n}^{*}$ onto the basis $\left\{\varphi_{0}, \ldots, \varphi_{n}\right\}$, i.e.,

$$
\gamma_{n, k}^{\left[\varphi^{*}\right]}:=\left\langle\varphi_{n}^{*}, \varphi_{k}\right\rangle_{\mathfrak{F}}, \quad k=0, \ldots, n
$$

Again, the zeros of a pORF $P_{n} \in{ }^{r} \mathcal{L}_{n}$ can be computed, either by means of the GEP (10), with

$$
\gamma_{n, k}^{[P]}:=\left\langle P_{n}, \varphi_{k}\right\rangle=A_{n}^{[P]} \delta_{n, k}+B_{n}^{[P]} \gamma_{n, k}^{\left[\varphi^{*}\right]}, \quad k=0, \ldots, n,
$$

or by means of the GEP (21) with the aid of the following theorem.

Theorem 14 A pORF $P_{n} \in \mathcal{L}_{n} \backslash \mathcal{L}_{n-1}$ is a qORF with coefficients

$$
A_{n}^{[Q]}=A_{n}^{[P]}+B_{n}^{[P]}\left\{\gamma_{n, n}^{\left[\varphi^{*}\right]}-\frac{\gamma_{n, n}^{[Z \varphi]} \gamma_{n, k}^{\left[\varphi^{*}\right]} / Z_{n-1}^{c}\left(\alpha_{n}\right)}{\gamma_{n, k}^{[Z \varphi]} / Z_{n-1}^{c}\left(\alpha_{n}\right)+\delta_{n-1, k}}\right\}, \quad k<n,
$$

and

$$
B_{n}^{[Q]}=B_{n}^{[P]} \frac{\gamma_{n, k}^{\left[*^{*}\right]}}{\gamma_{n, k}^{[Z \varphi]} / Z_{n-1}^{c}\left(\alpha_{n}\right)+\delta_{n-1, k}}, \quad k<n .
$$

Proof. The equalities directly follow from (18) and (27) by setting $\gamma_{n, k}^{[Q]}=\gamma_{n, k}^{[P]}$ for $k=0, \ldots, n$, and solving for $A_{n}^{[Q]}$ and $B_{n}^{[Q]}$.

Finally, to compute the corresponding coefficients $A_{n}^{[Q]}$ and $B_{n}^{[Q]}$ for a given pORF $P_{n} \in \mathcal{L}_{n} \backslash \mathcal{L}_{n-1}$, the expressions in the previous theorem require the computation of four inner products. The following two theorems, however, provide an alternative way to compute these corresponding coefficients.

Theorem 15 Let

$$
K(x):=\frac{\varphi_{n}^{*}(x)}{\varphi_{n}(x)}, \quad K_{\alpha}:=[K(x)]_{x=\alpha} \quad \text { and } \quad L(x):=\frac{Z_{n-1}^{c}(x) \varphi_{n}(x)}{Z_{n}(x) \varphi_{n-1}(x)} .
$$

Then a $p O R F P_{n} \in \mathcal{L}_{n} \backslash \mathcal{L}_{n-1}$ is a qORF with coefficients

$$
A_{n}^{[Q]}=A_{n}^{[P]}+B_{n}^{[P]} K_{\bar{\alpha}_{n-1}}
$$

and

$$
B_{n}^{[Q]}=\left[\left\{A_{n}^{[P]}+B_{n}^{[P]}\left(K(x)-K_{\bar{\alpha}_{n-1}}\right)\right\} L(x)\right]_{x=\alpha}, \quad \alpha \in \overline{\mathbb{C}} \backslash\left\{\bar{\alpha}_{n-1}\right\} .
$$


Proof. From Theorem 13 it follows that for every $x \in \overline{\mathbb{C}}$,

$$
\frac{P_{n}(x)}{\varphi_{n}(x)}=A_{n}^{[P]}+B_{n}^{[P]} \frac{\varphi_{n}^{*}(x)}{\varphi_{n}(x)}=A_{n}^{[Q]}+B_{n}^{[Q]} \frac{Z_{n}(x) \varphi_{n-1}(x)}{Z_{n-1}^{c}(x) \varphi_{n}(x)}=\frac{Q_{n}(x)}{\varphi_{n}(x)} .
$$

The equalities now easily follow.

Theorem 16 A pORF $P_{n} \in \mathcal{L}_{n} \backslash \mathcal{L}_{n-1}$ is a qORF with coefficients

$$
A_{n}^{[Q]}=A_{n}^{[P]}+\frac{B_{n}^{[P]} F_{n}}{E_{n}}\left\{\frac{1-G_{n} / Z_{n-1}^{c}\left(\alpha_{n-1}\right)}{1-D_{n} / Z_{n-1}^{c}\left(\alpha_{n-1}\right)}\right\}
$$

and

$$
B_{n}^{[Q]}=\frac{B_{n}^{[P]} F_{n}\left(G_{n}-D_{n}\right)}{1-D_{n} / Z_{n-1}^{c}\left(\alpha_{n-1}\right)}
$$

where $E_{n}$ and $D_{n}$ are defined as before in Theorem 1, and

$$
\begin{aligned}
& F_{n}=\left[\frac{\varphi_{n}^{*}(x)}{Z_{n}(x) \varphi_{n-1}(x)}\right]_{x=\alpha_{n-1}} \neq 0 \\
& \text { and } \quad G_{n}=\left[\frac{Z_{n-1}(x) \varphi_{n}^{*}(x)}{F_{n} Z_{n}(x) \varphi_{n-1}(x)}-Z_{n-1}(x)\right]_{x=\bar{\alpha}_{n-2}} .
\end{aligned}
$$

Proof. Since $\varphi_{n} \in{ }^{d} \mathcal{L}_{n}$, it follows that $\varphi_{n}^{*}$ is not degenerate and not exceptional. Hence, from (26) we deduce that $\varphi_{n}^{*}$ is of the form

$$
\varphi_{n}^{*}(x)=F_{n} Z_{n}(x)\left\{\left[1+\frac{G_{n}}{Z_{n-1}(x)}\right] \varphi_{n-1}(x)-\frac{H_{n}}{Z_{n-2}^{c}(x)} \varphi_{n-2}(x)\right\}, F_{n} \neq 0,
$$

where $F_{n}$ and $G_{n}$ are given by (29), and

$$
H_{n}=\frac{1-G_{n} / Z_{n-1}^{c}\left(\alpha_{n-1}\right)}{\bar{E}_{n-1}} \in \mathbb{C}_{0} .
$$

Consequently, the pORF $P_{n}$ satisfies

$$
P_{n}(x)=Z_{n}(x)\left\{\left[E_{n}^{[P]}+\frac{\hat{D}_{n}}{Z_{n-1}(x)}\right] \varphi_{n-1}(x)-\frac{\hat{C}_{n}}{Z_{n-2}^{c}(x)} \varphi_{n-2}(x)\right\},
$$

with

$$
E_{n}^{[P]}=A_{n}^{[P]} E_{n}+B_{n}^{[P]} F_{n}, \quad \hat{D}_{n}=A_{n}^{[P]} E_{n} D_{n}+B_{n}^{[P]} F_{n} G_{n}
$$

and

$$
\hat{C}_{n}=A_{n}^{[P]} E_{n} C_{n}+B_{n}^{[P]} F_{n} H_{n} .
$$

The equalities for $A_{n}^{[Q]}$ and $B_{n}^{[Q]}$ now follow by solving the system of equations

$$
\left\{\begin{array}{l}
E_{n}^{[Q]}=E_{n}^{[P]} \\
\tilde{D}_{n}=\hat{D}_{n}
\end{array}\right.
$$


where $E_{n}^{[Q]}$ and $\tilde{D}_{n}$ are given by (13) and (17) respectively. Finally, note that with $\tilde{C}_{n}$ and $A_{n}^{[Q]}$ given by (17) and (28) respectively, we indeed get that

$$
\tilde{C}_{n}=A_{n}^{[Q]} E_{n} C_{n}=A_{n}^{[P]} E_{n} C_{n}+B_{n}^{[P]} F_{n} H_{n}=\hat{C}_{n} .
$$

This ends the proof.

Remark 17 It is easily verified that $\frac{Z_{n}^{c}}{Z_{n}} \varphi_{n}^{*} \in \mathcal{L}_{n}^{\left[\bar{\alpha}_{n}\right]}$ is orthonormal on $\mathcal{L}_{n-1}$ with respect to the inner product (4). Moreover, since $\varphi_{n} \in{ }^{d} \mathcal{L}_{n}$, it follows that $\frac{Z_{n}^{c}}{Z_{n}} \varphi_{n}^{*} \in{ }^{d} \mathcal{L}_{n}^{\left[\bar{\alpha}_{n}\right]}$, so that the coefficients $F_{n}$ and $G_{n}$ can also be expressed in terms of inner products as in Theorem 1.

\section{Rational Gaussian quadrature}

As already pointed out at the beginning of Section 3, the main importance of qORFs is that, whenever their zeros are all real and simple, they can be used as nodes in a rational Gaussian quadrature formula. This is shown in the next theorem (for the proof we refer to [13, Thm. 2.3.5]).

Theorem 18 Suppose the zeros $x_{n, j}^{[Q]}$ of the $q O R F Q_{n} \in{ }^{r} \mathcal{L}_{n}$ are all real and simple, and let

$$
\lambda_{n, j}=\left\{\sum_{k=0}^{n-1}\left|\varphi_{k}\left(x_{n, j}^{[Q]}\right)\right|^{2}\right\}^{-1} .
$$

Then the quadrature

$$
\mathfrak{F}\{f\} \approx \sum_{j=1}^{n} \lambda_{n, j} f\left(x_{n, j}\right)=: \mathfrak{F}_{n}\{f\}
$$

is exact for every $f \in \mathcal{L}_{n-1} \cdot \mathcal{L}_{n-1}^{c}$. In the special case in which $Q_{n}(x)=$ $A_{n}^{[Q]} \varphi_{n}(x)$, the quadrature is exact for every $f \in \mathcal{L}_{n} \cdot \mathcal{L}_{n-1}^{c}$.

From (22) we deduce that the weights in (30) can be computed too, either by means of the GEP (10) with the projection coefficients $\gamma_{n, k}^{[Q]}$ given by (18), or by means of the GEP (21). The weights are then given by

$$
\lambda_{n, j}=\left|v_{n, j}^{[Q]}\right|^{2}\|1\|_{\mathfrak{F}}^{2},
$$

where $v_{n, j}^{[Q]}$ represents the first component of the eigenvector $\underline{v}_{n, j}^{[Q]}$.

The following theorem now provides a way to determine whether the zeros of a qORF $Q_{n} \in \mathcal{L}_{n} \backslash \mathcal{L}_{n-1}$ are all real and simple.

Theorem 19 The zeros of a $q O R F Q_{n} \in{ }^{d} \mathcal{L}_{n}$ are all real and simple iff the constant $v_{n}$, as defined in Theorem 7 , is equal to zero. 
Proof. Due to Theorem 7, $\frac{Z_{\alpha}}{Z_{n}} Q_{n} \in \mathcal{L}_{n}^{[\alpha]} \backslash \mathcal{L}_{n-1}$ is (up to a non-zero multiplicative factor) a nORF and has the same zeros as $Q_{n}$ whenever $\alpha \in \overline{\mathbb{C}}_{0}$ satisfies the conditions given in the statement of the theorem. This auxiliary pole $\alpha$ always exists and is not unique, but either it should lie in the upper half plane $\left(v_{n}>0\right)$, in the lower half plane $\left(v_{n}<0\right)$, or it should be in $\overline{\mathbb{R}}_{0}$ $\left(v_{n}=0\right)$. On the other hand, from Lemma 12 we know that the zeros of a nORF are all real and simple iff the last pole is in $\overline{\mathbb{R}}_{0}$. For this reason, the zeros of $Q_{n} \in{ }^{d} \mathcal{L}_{n}$ are all real and simple iff the auxiliary pole $\alpha \in \overline{\mathbb{R}}_{0}$, i.e., iff $v_{n}=0$. This concludes the proof.

Clearly, the zeros of a rational function $f_{n} \in \mathcal{L}_{n} \backslash \mathcal{L}_{n-1}$ are all real iff there exists a constant $\kappa_{n} \in \mathbb{T}$ so that $f_{n}^{*}(x)=\kappa_{n} f_{n}(x)$. So, from (23) it follows that the zeros of a pORF $P_{n} \in \mathcal{L}_{n} \backslash \mathcal{L}_{n-1}$ are all real iff either $\alpha_{n} \in \overline{\mathbb{R}}_{0}$ or $\tau_{n}^{[P]} \in \mathbb{T}$. Moreover, in [13, Lem. 2.3.4] it has been proved that the zeros of $P_{n}$, with $\tau_{n}^{[P]} \in \mathbb{T}$, are all simple. In particular this holds for $\alpha_{n} \in \overline{\mathbb{R}}_{0}$ (even if $\tau_{n}^{[P]} \notin \mathbb{T}$ ), due to the fact that the zeros of the pORF $P_{n}$ are then the zeros of the $\mathrm{nORF} \varphi_{n}$ (and hence, are independent of $\tau_{n}^{[P]}$ ).

Consider now the case of a positive bounded Borel measure $\mu$ with infinite support $\operatorname{supp}(\mu) \subseteq[a, b] \subsetneq \overline{\mathbb{R}}$, where the interval $[a, b]$ is assumed to be the smallest closed interval whose complement with respect to $\overline{\mathbb{C}}$ has $\mu$-measure zero, and suppose the poles $\alpha_{k}$ are bounded away from this interval. Without loss of generality we may assume $[a, b]=[-1,1]=: I$. Further, let the HPD linear functional $\mathfrak{F}$ be defined by

$$
\mathfrak{F}\{f\}=\int_{I} f(x) d \mu(x), \quad f \in \mathcal{L} .
$$

We then are concerned with the rational Gaussian quadrature formula (31) with $\left\{x_{n, j}^{[Q]}\right\}_{j=1}^{n} \subset I$. For sure, the zeros of the corresponding nORF $\varphi_{n}$ are all inside $I$ whenever $\alpha_{n} \in \overline{\mathbb{R}}_{I}$, so let us have a look now at the case in which $\alpha_{n} \notin \overline{\mathbb{R}}$. For $\tau_{n}^{[P]} \in \mathbb{T}$ it has been proved then in [37, Thm. 4.1] that at least $n-1$ zeros of $P_{n}$ are inside $(-1,1)$, and that there exist $\tau_{n}^{[P]} \in \mathbb{T}$ so that all the zeros of $P_{n}$ are in $I$. Thus, in order to have them all in $I$, it suffices that the argument $\theta_{n}$ of $\tau_{n}^{[P]}=e^{\mathbf{i} \theta_{n}}$ is inside an interval $\left[\theta_{b}, \theta_{e}\right]$, with $0<\theta_{e}-\theta_{b}<2 \pi$, where $\theta_{b}$ and $\theta_{e}$ are the arguments of $-\frac{\varphi_{n}( \pm 1)}{\varphi_{n}^{*}( \pm 1)}$ and $-\frac{\varphi_{n}(\mp 1)}{\varphi_{n}^{*}(\mp 1)}$ respectively, and the interval is chosen in such a way that the argument of $\left[-\frac{\varphi_{n}(x)}{\varphi_{n}^{*}(x)}\right]_{x=\infty}$ is not in the interval $\left[\theta_{b}, \theta_{e}\right]$.

As a consequence of Theorem 16 we can now prove the following.

Theorem 20 Let $\theta_{b}$ and $\theta_{e}$ be defined as above. Then, for $\alpha_{n} \notin \overline{\mathbb{R}}$, the zeros of a qORF $Q_{n} \in{ }^{d} \mathcal{L}_{n}$ are all in I and simple iff there exists a constant $\tau_{n}=$ $e^{\mathbf{i} \theta_{n}} \in \mathbb{T}$, with $\theta_{n} \in\left[\theta_{b}, \theta_{e}\right]$, so that

$$
D_{n}^{[Q]}=\frac{E_{n} D_{n}+F_{n} G_{n} \tau_{n}}{E_{n}+F_{n} \tau_{n}}
$$


Table 1 Definition of the Chebyshev weight functions $\mu^{\prime}(x)$ on $I$, respectively $\ddot{\mu}^{\prime}(z)$ on $\mathbb{T}$, and parameters $p$ and $q$ for $i=1,2,3$.

\begin{tabular}{|c|c|c|c|c|}
\hline$i$ & $\mu^{\prime}(x)$ & $\stackrel{\circ}{ }^{\prime}(z)$ & $p$ & $q$ \\
\hline 1 & $\left(1-x^{2}\right)^{-1 / 2}$ & $\frac{1}{\mathbf{i} z}$ & 1 & -1 \\
2 & $\left(\frac{1-x}{1+x}\right)^{1 / 2}$ & $\frac{-(z-1)^{2}}{2 \mathbf{i} z^{2}}$ & 1 & 1 \\
3 & $\left(1-x^{2}\right)^{1 / 2}$ & $\frac{-\left(z^{2}-1\right)^{2}}{4 \mathbf{i} z^{3}}$ & 2 & 1 \\
\hline
\end{tabular}

Whenever $\alpha_{n} \in \overline{\mathbb{R}}_{I}$, the equality reduces to $D_{n}^{[Q]}=D_{n}$ so that the statement still holds under the condition that 'iff' is replaced with 'if'.

Proof. From (14) we deduce that

$$
D_{n}^{[Q]}=\frac{E_{n} D_{n}+\tau_{n}^{[Q]}}{E_{n}+\tau_{n}^{[Q]} / Z_{n-1}^{c}\left(\alpha_{n-1}\right)},
$$

while it follows from Theorem 16 that

$$
\tau_{n}^{[Q]}=\frac{E_{n} F_{n}\left(G_{n}-D_{n}\right) \tau_{n}^{[P]}}{E_{n}\left(1-D_{n} / Z_{n-1}^{c}\left(\alpha_{n-1}\right)\right)+F_{n}\left(1-G_{n} / Z_{n-1}^{c}\left(\alpha_{n-1}\right)\right) \tau_{n}^{[P]}} .
$$

The statement now follows by plugging this equality for $\tau_{n}^{[Q]}$ into (33) and simplifying.

We conclude this section with summarizing the different steps in the construction of the rational Gaussian quadrature formula (31):

1. Construct the GEP (9).

2. For a given parameter $\tau_{n} \in \overline{\mathbb{C}}$ in the $n$th qORF (or pORF):

(a) check whether the qORF (or pORF) is regular;

(b) check whether its zeros are all real, and in the interval $[-1,1]$ (if necessary).

3. Construct the corresponding modified GEP (10) or (21).

4. Solve the modified GEP to obtain the nodes and weights in the quadrature formula.

\section{Illustrative example}

To illustrate the results of the previous sections, we consider the case in which the measure $\mu$ in (32) is one of the Chebyshev weight functions defined in Table 1. Given a sequence of complex numbers $\mathcal{B}=\left\{\beta_{1}, \beta_{2}, \ldots\right\} \subset \mathbb{D}$, we define the Blaschke factors for $\mathcal{B}$ as

$$
\zeta_{k}(z):=\zeta_{\beta_{k}}(z)=\frac{z-\beta_{k}}{1-\bar{\beta}_{k} z}, \quad k=1,2, \ldots,
$$


and the corresponding Blaschke products for $\mathcal{B}$ as

$$
B_{0}(z) \equiv 1, \quad B_{k}(z)=\zeta_{k}(z) B_{k-1}(z), \quad k=1,2, \ldots .
$$

Further, we denote the Joukowski Transformation $x=\frac{1}{2}\left(z+z^{-1}\right)$ by $x=J(z)$, mapping the open unit disc $\mathbb{D}$ onto the cut Riemann sphere $\overline{\mathbb{C}}_{I}$ and the unit circle $\mathbb{T}$ onto the interval $I$. When $z=e^{\mathrm{i} \theta}$, then $x=J(z)=\cos \theta$. In what follows we will assume that $x$ and $z$ are related by this transformation. The inverse mapping is denoted by $z=J^{\text {inv }}(x)$ and is chosen so that $z \in \mathbb{D}$ if $x \in$ $\overline{\mathbb{C}}_{I}$. With the sequence $\mathcal{A}=\left\{\alpha_{1}, \alpha_{2}, \ldots\right\} \subset \overline{\mathbb{C}}_{I}$ we then associate a sequence $\mathcal{B}=\left\{\beta_{1}, \beta_{2}, \ldots\right\} \subset \mathbb{D}$, so that $\beta_{k}=J^{\text {inv }}\left(\alpha_{k}\right)$, and set $\beta_{0}=J^{\text {inv }}\left(\alpha_{0}\right)=0$.

Explicit expressions for the so-called Chebyshev nORFs, related to the $i$ th weight function in Table 1, with arbitrary complex poles outside I are now given in the next theorem. For the proof we refer to [18, Thm. 3.2].

Theorem 21 Let $x=J(z) \in \overline{\mathbb{C}}$ and $\alpha_{j}=J\left(\beta_{j}\right) \in \overline{\mathbb{C}}_{I}, j=1, \ldots, k$. Suppose $p$ and $q$ are defined in terms of $i$ by Table 1 and let $N_{k}$ represent the normalization factor given by

$$
N_{k}=\sqrt{\frac{2^{i}}{\pi}} \sqrt{1-\left|\beta_{k}\right|^{2}}
$$

Then the Chebyshev nORFs $\varphi_{k}(x), k \geqslant 0$, related to the inner product (4), with the HPD linear functional $\mathfrak{F}$ defined by (32), and $i$-th weight function in Table 1, are given by

$\varphi_{0}(x) \equiv \sqrt{\frac{p}{\pi}}, \quad \varphi_{k}(x)=\frac{q N_{k}}{2 z^{i-1}+q-3}\left(\frac{z^{i} B_{k-1}^{c}(z)}{1-\beta_{k} z}-\frac{q}{\left(z-\beta_{k}\right) B_{k-1}(z)}\right), k \geqslant 1$.

In what follows, we call a qORF or a pORF based on the nORFs $\varphi_{k}$ with respect to the $i$-th weight function in Table 1 a Chebyshev qORF or a Chebyshev pORF. In the remainder $i$ will consistently refer to the values 1,2 or 3 , while $p$ and $q$ will refer to the corresponding values in Table 1 .

We will also need the following lemmas. The first lemma has been proved in [18], while the second lemma has been proved in [39, Lem. 3.3].

Lemma 22 Let $x=J(z) \in \overline{\mathbb{C}}$ and $\alpha_{k}=J\left(\beta_{k}\right) \in \overline{\mathbb{C}}_{I}$. Then

$$
Z_{k}(x)=\varsigma_{k} \frac{z^{2}+1}{\left(z-\beta_{k}\right)\left(1-\beta_{k} z\right)}, \quad \varsigma_{k}=\frac{1+\beta_{k}^{2}}{2},
$$

and

$$
\frac{Z_{k}(x)}{Z_{k}^{c}(x)}=\sigma_{k} \frac{\zeta_{k}^{c}(z)}{\zeta_{k}(z)}, \quad \sigma_{k}=\frac{\varsigma_{k}}{\bar{\varsigma}_{k}} \in \mathbb{T} .
$$

Lemma 23 Whenever $z \in \mathbb{T}$, it holds for every $k>0$ that the derivative $B_{k}^{\prime}(z)$ can be written as

$$
B_{k}^{\prime}(z)=\frac{B_{k}(z)}{z} \sum_{j=1}^{k} \frac{1-\left|\beta_{k}\right|^{2}}{\left|z-\beta_{k}\right|^{2}} .
$$


Taking by convention that an empty sum equals zero, the equality holds for $k=0$ too.

In the case of the Chebyshev nORFs, explicit expressions are known for the recurrence coefficients $E_{k}$ and $D_{k}$ in Theorem 1 . For $k>1$ they are given by (see [32, Sec. 4] and [39, Thm. 3.5])

$$
E_{k}=\frac{2 \sqrt{\left(1-\left|\beta_{k}\right|^{2}\right)\left(1-\left|\beta_{k-1}\right|^{2}\right)}\left(1-\beta_{k} \beta_{k-1}\right)}{\left(1+\beta_{k}^{2}\right)\left(1+\beta_{k-1}^{2}\right)}
$$

and

$$
\begin{aligned}
D_{k}=-\frac{1+\beta_{k-1}^{2}}{2\left(1-\left|\beta_{k-1}\right|^{2}\right)} \times & \\
& \frac{\left(1-\left|\beta_{k-1}\right|^{2}\right)\left(\beta_{k}+\bar{\beta}_{k-2}\right)+2 \Re\left\{\beta_{k-1}\right\}\left(1-\beta_{k} \bar{\beta}_{k-2}\right)}{\left(1-\beta_{k} \beta_{k-1}\right)\left(1-\beta_{k-1} \bar{\beta}_{k-2}\right)},
\end{aligned}
$$

with $\beta_{0}=0$. For $k=1$ they are given by

$$
E_{1}=\sqrt{\frac{2^{i}}{p}} \frac{\sqrt{1-\left|\beta_{1}\right|^{2}}}{1+\beta_{1}^{2}}
$$

and

$$
D_{1}=\frac{p}{2^{i-1}}\left(1-i \bmod 2-\beta_{1}\right)
$$

From Theorem 21 and Lemma 22 it follows for $n>0$ that

$$
\begin{aligned}
\varphi_{n}^{*}(x) & =\frac{q N_{n}}{2 z^{i-1}+q-3} \nu_{n} \frac{B_{n}^{c}(z)}{B_{n}(z)}\left(\frac{z^{i} B_{n-1}(z)}{1-\bar{\beta}_{n} z}-\frac{q}{\left(z-\bar{\beta}_{n}\right) B_{n-1}^{c}(z)}\right) \\
& =\nu_{n} \frac{q N_{n}}{2 z^{i-1}+q-3}\left(\frac{z^{i} B_{n-1}^{c}(z)\left[\frac{z-\bar{\beta}_{n}}{z-\beta_{n}}\right]}{1-\beta_{n} z}-\frac{q\left[\frac{1-\bar{\beta}_{n} z}{1-\beta_{n} z}\right]}{\left(z-\beta_{n}\right) B_{n-1}(z)}\right),(
\end{aligned}
$$

where

$$
\nu_{n}=\prod_{j=1}^{n} \sigma_{k} \in \mathbb{T}
$$

Further, note that

$$
\frac{Z_{n}^{c}(x)}{Z_{n}(x)} \varphi_{n}^{*}(x)=\nu_{n-1} \frac{q N_{n}}{2 z^{i-1}+q-3}\left(\frac{z^{i} B_{n-1}^{c}(z)}{1-\beta_{n} z}-\frac{q}{\left(z-\beta_{n}\right) B_{n-1}(z)}\right),
$$

so that the recurrence coefficients $F_{n}$ and $G_{n}$, defined as before in Theorem 16 , are given by

$$
F_{n}=\nu_{n} E_{n} \frac{1-\bar{\beta}_{n} \beta_{n-1}}{1-\beta_{n} \beta_{n-1}}
$$


respectively

$$
G_{1}=\frac{p}{2^{i-1}}\left(1-i \bmod 2-\bar{\beta}_{1}\right)
$$

and

$$
\begin{aligned}
G_{n}=-\frac{1+\beta_{n-1}^{2}}{2\left(1-\left|\beta_{n-1}\right|^{2}\right)} \times & \\
& \quad \frac{\left(1-\left|\beta_{n-1}\right|^{2}\right)\left(\bar{\beta}_{n}+\bar{\beta}_{n-2}\right)+2 \Re\left\{\beta_{n-1}\right\}\left(1-\bar{\beta}_{n} \bar{\beta}_{n-2}\right)}{\left(1-\bar{\beta}_{n} \beta_{n-1}\right)\left(1-\beta_{n-1} \bar{\beta}_{n-2}\right)}, \quad n>1 .
\end{aligned}
$$

The following theorem now provides expressions to check whether a given Chebyshev qORF or Chebyshev pORF is in ${ }^{r} \mathcal{L}_{n}$.

Theorem 24 Let $x=J(z) \in \overline{\mathbb{C}}$ and $\alpha_{k}=J\left(\beta_{k}\right) \in \overline{\mathbb{C}}_{I}, k=0, \ldots, n$, with $\beta_{0}=0$. Further, let $\varepsilon_{n}$ for $n>0$ be defined by

$$
\varepsilon_{n}= \begin{cases}\frac{\sqrt{2}}{2}\left(1-\beta_{n}^{2}\right), & i=1 \\ \left(1+\beta_{n}\right), & i=2 \\ 1, & i=3\end{cases}
$$

Then a Chebyshev $q O R F Q_{n}$ with respect to the $i$-th weight function in Table 1 is in ${ }^{r} \mathcal{L}_{n}$ iff $\tau_{n}^{[Q]} \in \mathbb{C}$ and

$$
\tau_{1}^{[Q]} \neq-\frac{2 \sqrt{1-\left|\beta_{1}\right|^{2}}}{\beta_{1}\left(1+\beta_{1}^{2}\right)} \varepsilon_{1}
$$

respectively

$$
\begin{aligned}
\tau_{n}^{[Q]} & \neq-\frac{\bar{\varsigma}_{n-1} N_{n}\left(1-\beta_{n} \beta_{k}\right)}{\varsigma_{n} N_{n-1}\left(\beta_{k}-\bar{\beta}_{n-1}\right)} \times \\
& \left\{1-q \beta_{k}^{i}\left(\frac{\beta_{k}-\beta_{n-1}}{1-\beta_{n-1} \beta_{k}}\right) B_{n-2}\left(\beta_{k}\right) B_{n-2}^{c}\left(\beta_{k}\right)\right\}^{-1}, n>1, k=0, \ldots, n .
\end{aligned}
$$

On the other hand, a Chebyshev pORF $P_{n}$ with respect to the $i$-th weight function in Table 1 is in ${ }^{r} \mathcal{L}_{n}$ iff

$$
\tau_{n}^{[P]} \neq-\bar{\nu}_{n} \frac{1-\beta_{n} \bar{\beta}_{n-1}}{1-\bar{\beta}_{n} \bar{\beta}_{n-1}}, n>0
$$

and

$$
\tau_{n}^{[P]} \neq-\bar{\nu}_{n}\left\{\frac{1-\bar{\beta}_{n} \beta_{k}}{1-\beta_{n} \beta_{k}}-q \beta_{k}^{i} B_{n}^{c}\left(\beta_{k}\right) B_{n-1}\left(\beta_{k}\right)\right\}^{-1}, n>0, k=0, \ldots, n,
$$

where $\nu_{n} \in \mathbb{T}$ is given by (38). 
Proof. By computing $\left[-\frac{Z_{n-1}^{c}(x) \varphi_{n}(x)}{Z_{n}(x) \varphi_{n-1}(x)}\right]_{x=\alpha_{k}}$, for $n>0$ and $k=0, \ldots, n$, with the aid of Theorem 21 and Lemma 22, the inequalities for $\tau_{n}^{[Q]}$ directly follow. The inequalities for $\tau_{n}^{[P]}$ easily follow by computing $\left[-\frac{\varphi_{n}(x)}{\varphi_{n}^{*}(x)}\right]_{x=\alpha}$, for $n>0$ with $\alpha=\bar{\alpha}_{n-1}$ or $\alpha=\alpha_{k}, k=0, \ldots, n$, by means of (37) and Theorem 21 .

Next, the following theorem provides expressions to check whether the zeros of a given Chebyshev pORF $P_{n}$, for which $\tau_{n}^{[P]} \in \mathbb{T}$, are all in $I$.

Theorem 25 Let $x=J(z) \in \overline{\mathbb{C}}$ and $\alpha_{k}=J\left(\beta_{k}\right) \in \overline{\mathbb{C}}_{I}$, for $k=1, \ldots, n$ and $n \geqslant 1$. Suppose $\tau_{n}^{[P]} \in \mathbb{T}$ and define

$$
\rho_{n}=\tau_{n}^{[P]} \nu_{n} \in \mathbb{T}
$$

where $\nu_{n} \in \mathbb{T}$ is given by (38). Further, let $\Phi_{n}^{ \pm}$be given by

$$
\Phi_{n}^{ \pm}=\sum_{k=1}^{n-1} \frac{1-\left|\beta_{k}\right|^{2}}{\left|1 \pm \beta_{k}\right|^{2}}
$$

Then a Chebyshev pORF $P_{n}$ with respect to the $i$-th weight function in Table 1 has a zero

1. at infinity iff $\rho_{n}=\rho_{n}^{[\infty]}:=-1$;

2. in $x=-1$ iff

$$
\rho_{n}=\rho_{n}^{[i-]}:= \begin{cases}-\frac{1+\beta_{n}}{1+\bar{\beta}_{n}}, & i=1 \text { or } 2 \\ -\frac{1+\beta_{n}+\frac{1}{1+\Phi_{n}^{+}}}{1+\bar{\beta}_{n}+\frac{1}{1+\Phi_{n}^{+}}}, & i=3\end{cases}
$$

3. in $x=1$ iff

$$
\rho_{n}=\rho_{n}^{[i+]}:= \begin{cases}-\frac{1-\beta_{n}}{1-\bar{\beta}_{n}}, & i=1 \\ -\frac{1-\beta_{n}+\frac{2}{1+2 \Phi_{n}^{-}}}{1-\bar{\beta}_{n}+\frac{2}{1+2 \Phi_{n}^{-}}}, & i=2 \\ -\frac{1-\beta_{n}+\frac{1}{1+\Phi_{n}^{-}}}{1-\bar{\beta}_{n}+\frac{1}{1+\Phi_{n}^{-}}}, & i=3 .\end{cases}
$$

Proof. The zero at infinity immediately follows from (40) with $k=0$. For a zero in $x= \pm 1$ (and hence, in $z= \pm 1$ ), it should hold that

$$
\rho_{n}=-\left[\frac{z^{i}\left(z-\beta_{n}\right) B_{n-1}(z) B_{n-1}^{c}(z)-q\left(1-\beta_{n} z\right)}{z^{i}\left(z-\bar{\beta}_{n}\right) B_{n-1}(z) B_{n-1}^{c}(z)-q\left(1-\bar{\beta}_{n} z\right)}\right]_{z= \pm 1} .
$$

Consequently, taking into account that $B_{n-1}( \pm 1) B_{n-1}^{c}( \pm 1)=1$, we obtain for $i=1$ that

$$
\rho_{n}^{[1+]}=-\frac{1-\beta_{n}}{1-\bar{\beta}_{n}} \quad \text { and } \quad \rho_{n}^{[1-]}=-\frac{1+\beta_{n}}{1+\bar{\beta}_{n}}
$$


Next, consider the case in which $i=2$. For $x=-1=z$ we then obtain the same result, i.e., $\rho_{n}^{[2-]}=\rho_{n}^{[1-]}$. For $x=1=z$, on the other hand, using l'Hôpital we get that

$$
\rho_{n}^{[2+]}=-\frac{\left(3-\beta_{n}\right)+\left(1-\beta_{n}\right)\left[\left(B_{n-1}(z) B_{n-1}^{c}(z)\right)^{\prime}\right]_{z=1}}{\left(3-\bar{\beta}_{n}\right)+\left(1-\bar{\beta}_{n}\right)\left[\left(B_{n-1}(z) B_{n-1}^{c}(z)\right)^{\prime}\right]_{z=1}},
$$

where it follows from (34) that

$$
\left[\left(B_{n-1}(z) B_{n-1}^{c}(z)\right)^{\prime}\right]_{z=1}=2 \Phi_{n}^{-} .
$$

Finally, for $i=3$ we get for $x=1=z$ that

$$
\rho_{n}^{[3+]}=-\frac{2\left(2-\beta_{n}\right)+\left(1-\beta_{n}\right)\left[\left(B_{n-1}(z) B_{n-1}^{c}(z)\right)^{\prime}\right]_{z=1}}{2\left(2-\beta_{n}\right)+\left(1-\bar{\beta}_{n}\right)\left[\left(B_{n-1}(z) B_{n-1}^{c}(z)\right)^{\prime}\right]_{z=1}},
$$

while we get for $x=-1=z$ that

$$
\rho_{n}^{[3+]}=-\frac{2\left(2+\beta_{n}\right)-\left(1+\beta_{n}\right)\left[\left(B_{n-1}(z) B_{n-1}^{c}(z)\right)^{\prime}\right]_{z=-1}}{2\left(2+\beta_{n}\right)-\left(1+\bar{\beta}_{n}\right)\left[\left(B_{n-1}(z) B_{n-1}^{c}(z)\right)^{\prime}\right]_{z=-1}},
$$

where it follows from (34) that

$$
\left[\left(B_{n-1}(z) B_{n-1}^{c}(z)\right)^{\prime}\right]_{z=-1}=-2 \Phi_{n}^{+} .
$$

Let $P_{n}(x)=A_{n}^{[P]}\left[\varphi_{n}(x)+\tau_{n}^{[P]} \varphi_{n}^{*}(x)\right]=Q_{n}(x) \in{ }^{r} \mathcal{L}_{n}$, with $\rho_{n} \in \mathbb{T} \backslash\{-1\}$, where $\rho_{n}$ is given by $(41)$, and define

$$
\tilde{\beta}_{n}=\frac{\beta_{n}+\rho_{n} \bar{\beta}_{n}}{1+\rho_{n}}
$$

In $[18$, Sec. 4$]$ it has been proved then that, whenever $\tilde{\beta}_{n} \in(-1,1)$ and $\tilde{\alpha}_{n}=$ $J\left(\tilde{\beta}_{n}\right) \in \overline{\mathbb{C}}_{I}$, the rational function $\frac{Z_{\tilde{\alpha}_{n}}}{Z_{n}} Q_{n} \in \mathcal{L}_{n}^{\left[\tilde{\alpha}_{n}\right]}$ is orthogonal with respect to the HPD linear functional $\mathfrak{G}=\mathfrak{F}$. Consequently, the zeros of a Chebyshev qORF $Q_{n}$ are for sure all in $I$ and simple if its recurrence coefficient $D_{n}^{[Q]}$ is of the form

$$
D_{1}^{[Q]}=\frac{p}{2^{i-1}}\left(1-i \bmod 2-\tilde{\beta}_{1}\right),
$$

respectively

$$
\begin{aligned}
D_{n}^{[Q]}= & -\frac{1+\beta_{n-1}^{2}}{2\left(1-\left|\beta_{n-1}\right|^{2}\right)} \times \\
& \frac{\left(1-\left|\beta_{n-1}\right|^{2}\right)\left(\tilde{\beta}_{n}+\bar{\beta}_{n-2}\right)+2 \Re\left\{\beta_{n-1}\right\}\left(1-\tilde{\beta}_{n} \bar{\beta}_{n-2}\right)}{\left(1-\tilde{\beta}_{n} \beta_{n-1}\right)\left(1-\beta_{n-1} \bar{\beta}_{n-2}\right)}, \quad n>1,
\end{aligned}
$$


with $\beta_{0}=0$ and $\tilde{\beta}_{n} \in I$. So, let us now define $\beta_{n}^{[i \pm]}$ as

$$
\beta_{n}^{[i-]}:= \begin{cases}-1, & i=1 \text { or } 2 \\ -1-\frac{1}{1+\Phi_{n}^{+}}, & i=3\end{cases}
$$

and

$$
\beta_{n}^{[i+]}:= \begin{cases}1, & i=1 \\ 1+\frac{2}{1+2 \Phi_{n}^{-}}, & i=2 \\ 1+\frac{1}{1+\Phi_{n}^{-}}, & i=3\end{cases}
$$

i.e.,

$$
\beta_{n}^{[i \pm]}=\frac{\beta_{n}+\rho_{n}^{[i \pm]} \bar{\beta}_{n}}{1+\rho_{n}^{[i \pm]}} \quad \text { if } \quad \beta_{n} \in \mathbb{D}_{I} .
$$

Then for $\beta_{n} \in \mathbb{D}_{I}$ we deduce from the proof of Theorem 20 that the zeros of a Chebyshev qORF $Q_{n}$ are all in $I$ and simple iff its recurrence coefficient $D_{n}^{[Q]}$ is of the form given above, with $\tilde{\beta}_{n} \in\left[\beta_{n}^{[i-]}, \beta_{n}^{[i+]}\right]$. Further, since the $\beta_{n}^{[i \pm]}$ are independent of $\beta_{n}$, the same holds true for $\beta_{n} \in I$.

The following theorem now gives a simple relation between the coefficients $A_{n}^{[P]}$ and $B_{n}^{[P]}$ of a given Chebyshev pORF and the coefficients $A_{n}^{[Q]}$ and $B_{n}^{[Q]}$ of the corresponding Chebyshev qORF.

Theorem 26 Let $x=J(z) \in \overline{\mathbb{C}}$ and $\alpha_{k}=J\left(\beta_{k}\right) \in \overline{\mathbb{C}}_{I}, k=0, \ldots, n$, with $\beta_{0}=0$. Consider the Chebyshev pORF $P_{n}(x)=A_{n}^{[P]} \varphi_{n}(x)+B_{n}^{[P]} \varphi_{n}^{*}(x) \in$ $\mathcal{L}_{n} \backslash \mathcal{L}_{n-1}$ with respect to the $i$-th weight function in Table 1 , and let $e_{n}$ be defined by

$$
e_{n}= \begin{cases}\sqrt{2}, & (n, i)=(1,1) \\ 1, & \text { otherwise }\end{cases}
$$

Then the corresponding coefficients $A_{n}^{[Q]}$ and $B_{n}^{[Q]}$ are given by

$$
A_{n}^{[Q]}=A_{n}^{[P]}+B_{n}^{[P]} \nu_{n} \frac{1-\bar{\beta}_{n} \bar{\beta}_{n-1}}{1-\beta_{n} \bar{\beta}_{n-1}}
$$

and

$$
B_{n}^{[Q]}=B_{n}^{[P]} \nu_{n} \sqrt{\frac{1-\left|\beta_{n}\right|^{2}}{1-\left|\beta_{n-1}\right|^{2}}} \frac{\mathrm{i} 2 \Im\left\{\beta_{n}\right\}\left(1+\bar{\beta}_{n-1}^{2}\right)}{\left(1-\beta_{n} \bar{\beta}_{n-1}\right)\left(1+\beta_{n}^{2}\right)} e_{n},
$$

where $\nu_{n}$ is defined as before in (38). Thus, for $\tau_{n}^{[P]} \in \mathbb{T}$, it holds that

$$
\tau_{n}^{[Q]}=\varpi_{n} \sqrt{\frac{1-\left|\beta_{n}\right|^{2}}{1-\left|\beta_{n-1}\right|^{2}}}\left(\frac{1+\bar{\beta}_{n-1}^{2}}{1+\beta_{n}^{2}}\right) e_{n},
$$

where

$$
\varpi_{n}=\left\{\begin{array}{cl}
\left(\frac{\rho_{n}}{1+\rho_{n}}\right) \mathbf{i} 2 \Im\left\{\beta_{n}\right\}\left(1-\bar{\beta}_{n-1} \tilde{\beta}_{n}\right)^{-1}, & \rho_{n} \neq-1 \\
1 / \bar{\beta}_{n-1}, & \rho_{n}=-1,
\end{array}\right.
$$

and $\rho_{n}$ and $\tilde{\beta}_{n}$ are given by (41) and (42). 
Proof. The equality for $A_{n}^{[Q]}$ directly follows from Theorems 15 and 24 .

Next, suppose $\beta_{n-1} \in \mathbb{D}_{I}$. Theorem 16 together with (39) yields

$$
A_{n}^{[Q]}=A_{n}^{[P]}+B_{n}^{[P]} \nu_{n} \frac{1-\bar{\beta}_{n} \beta_{n-1}}{1-\beta_{n} \beta_{n-1}}\left\{\frac{1-G_{n} / Z_{n-1}^{c}\left(\alpha_{n-1}\right)}{1-D_{n} / Z_{n-1}^{c}\left(\alpha_{n-1}\right)}\right\},
$$

and hence,

$$
1-G_{n} / Z_{n-1}^{c}\left(\alpha_{n-1}\right)=\left(1-D_{n} / Z_{n-1}^{c}\left(\alpha_{n-1}\right)\right)\left|\frac{1-\beta_{n} \beta_{n-1}}{1-\beta_{n} \bar{\beta}_{n-1}}\right|^{2},
$$

or equivalently,

$$
\left(G_{n}-D_{n}\right) / Z_{n-1}^{c}\left(\alpha_{n-1}\right)=\left(1-D_{n} / Z_{n-1}^{c}\left(\alpha_{n-1}\right)\right)\left\{1-\left|\frac{1-\beta_{n} \beta_{n-1}}{1-\beta_{n} \bar{\beta}_{n-1}}\right|^{2}\right\} .
$$

Thus

$$
B_{n}^{[Q]}=B_{n}^{[P]} \nu_{n} \frac{\left(\frac{1-\bar{\beta}_{n} \beta_{n-1}}{1-\beta_{n} \beta_{n-1}}-\frac{1-\bar{\beta}_{n} \bar{\beta}_{n-1}}{1-\beta_{n} \bar{\beta}_{n-1}}\right) E_{n}}{1 / Z_{n-1}^{c}\left(\alpha_{n-1}\right)} .
$$

The equality in (44) now easily follows.

Since for $\beta_{n} \in I$ it should hold that $B_{n}^{[Q]}=0$, it follows that the equality in (44) remains valid for $\beta_{n-1} \in I$ whenever $\beta_{n} \in I$ too. So, consider now the case in which $\beta_{n-1} \in I$ and $\beta_{n} \in \mathbb{D}_{I}$. From Theorem 16 and (39) we then deduce that

$$
B_{n}^{[Q]}=B_{n}^{[P]} \nu_{n} E_{n} \frac{1-\bar{\beta}_{n} \beta_{n-1}}{1-\beta_{n} \beta_{n-1}}\left(G_{n}-D_{n}\right),
$$

with

$$
G_{1}-D_{1}=\frac{p}{2^{i-1}} 2 \mathbf{i} \Im\left\{\beta_{1}\right\}
$$

and

$$
G_{n}-D_{n}=\frac{\mathbf{i} \Im\left\{\beta_{n}\right\}\left(1+\beta_{n-1}^{2}\right)^{2}}{\left|1-\beta_{n} \beta_{n-1}\right|^{2}\left(1-\left|\beta_{n-1}\right|^{2}\right)} .
$$

Also now the equality in (44) easily follows.

Finally, in order to compute the zeros of a Chebyshev qORF or Chebyshev pORF by means of the GEP (10), we need expressions for the projection coefficients $\gamma_{n, k}^{[Z \varphi]}$ or $\gamma_{n, k}^{\left[\varphi^{*}\right]}, k=0, \ldots, n$.

Theorem 27 Let $x=J(z) \in \overline{\mathbb{C}}$ and $\alpha_{k}=J\left(\beta_{k}\right) \in \overline{\mathbb{C}}_{I}, k=1, \ldots, n$. Suppose the $n$ ORFs $\varphi_{k}, k=0, \ldots, n$, with respect to the $i$-th weight function in Table 1 are defined as before in Theorem 21. Then the projection coefficients $\gamma_{n, k}^{\left[\varphi^{*}\right]}$ of $\varphi_{n}^{*}, n>0$, onto the basis $\left\{\varphi_{0}, \ldots, \varphi_{n}\right\}$ are given by

$$
\gamma_{n, 0}^{\left[\varphi^{*}\right]}=\frac{\nu_{n}}{\varepsilon_{n}} \mathbf{i} 2 \Im\left\{\beta_{n}\right\} \sqrt{1-\left|\beta_{n}\right|^{2}} B_{n-1}^{c}\left(\beta_{n}\right),
$$


respectively

$$
\begin{aligned}
\gamma_{n, k}^{\left[\varphi^{*}\right]}=\nu_{n} \sqrt{\left(1-\left|\beta_{n}\right|^{2}\right)\left(1-\left|\beta_{k}\right|^{2}\right)} \frac{\mathbf{i} 2 \Im\left\{\beta_{n}\right\} B_{n-1}^{c}\left(\beta_{n}\right)}{\left(1-\beta_{n}^{2}\right)\left(1-\beta_{n} \bar{\beta}_{k}\right) B_{k}^{c}\left(\beta_{n}\right)} \times & \\
& \left\{\frac{1-\beta_{n} \bar{\beta}_{k}}{1-\beta_{n} \beta_{k}}-q \beta_{n}^{i} B_{k}^{c}\left(\beta_{n}\right) B_{k-1}\left(\beta_{n}\right)\right\}, \quad k=1, \ldots, n,
\end{aligned}
$$

where $\nu_{n} \in \mathbb{T}$ is given by (38), and $\varepsilon_{n}$ is defined as before in Theorem 24. The expression for $k=n$ can be simplified as follows:

$$
\gamma_{n, n}^{\left[\varphi^{*}\right]}=\frac{\nu_{n}}{1-\beta_{n}^{2}}\left\{\left(1-\left|\beta_{n}\right|^{2}\right)-q \mathbf{i} 2 \Im\left\{\beta_{n}\right\} \beta_{n}^{i} B_{n-1}^{c}\left(\beta_{n}\right) B_{n-1}\left(\beta_{n}\right)\right\}
$$

Proof. First, note that (see [18])

$$
\int_{I} \mu^{\prime}(x) d x=\frac{1}{2} \oint_{\mathbb{T}} \frac{-q}{2 \mathbf{i}(2 z)^{i}}\left\{\frac{q}{2 z^{i-1}+q-3}\right\}^{-2} d z=\frac{1}{2} \oint_{\mathbb{T}} \stackrel{\mu}{ }^{\prime}(z) d z,
$$

where $\mu^{\prime}(z)$ is given in Table 1 , and that for any function $f$ it holds that

$$
\oint_{\mathbb{T}}\{f(z)+f(1 / z)\} \stackrel{\circ}{\mu}^{\prime}(z) d z=2 \oint_{\mathbb{T}} f(z) \dot{\mu}^{\prime}(z) d z
$$

Consequently, for $k=0$ we obtain that

$$
\begin{aligned}
\gamma_{n, 0}^{\left[\varphi^{*}\right]} & =-\frac{\nu_{n} N_{n} \varphi_{0}}{2 \mathbf{i} \cdot 2^{i}} \oint_{\mathbb{T}} \frac{\left(z-\bar{\beta}_{n}\right) B_{n-1}^{c}(z)}{\left(z-\beta_{n}\right)\left(1-\beta_{n} z\right)}\left[2 z^{i-1}+q-3\right] d z \\
& =-\nu_{n} \sqrt{\frac{p}{2^{i}}} \sqrt{1-\left|\beta_{n}\right|^{2}} \operatorname{Res}\left\{\frac{\left(z-\bar{\beta}_{n}\right) B_{n-1}^{c}(z)}{\left(z-\beta_{n}\right)\left(1-\beta_{n} z\right)}\left[2 z^{i-1}+q-3\right], z=\beta_{n}\right\} \\
& =-\nu_{n} \sqrt{\frac{p}{2^{i}}} \sqrt{1-\left|\beta_{n}\right|^{2}} \frac{\mathbf{i} 2 \Im\left\{\beta_{n}\right\} B_{n-1}^{c}\left(\beta_{n}\right)}{1-\beta_{n}^{2}}\left[2 \beta_{n}^{i-1}+q-3\right]
\end{aligned}
$$

where it is easily verified that

$$
-\sqrt{\frac{p}{2^{i}}}\left(\frac{2 \beta_{n}^{i-1}+q-3}{1-\beta_{n}^{2}}\right)=\varepsilon_{n}^{-1}
$$


For $k>0$, on the other hand, we obtain that

$$
\begin{aligned}
& \gamma_{n, k}^{\left[\varphi^{*}\right]}= \frac{q \nu_{n} N_{n} N_{k}}{2 \mathbf{i} \cdot 2^{i}} \times \\
& \oint_{\mathbb{T}} \frac{\left(z-\bar{\beta}_{n}\right) B_{n-1}^{c}(z)}{\left(z-\beta_{n}\right)\left(1-\beta_{n} z\right)}\left(\frac{z^{i} B_{k-1}(z)}{1-\bar{\beta}_{k} z}-\frac{q}{\left(z-\bar{\beta}_{k}\right) B_{k-1}^{c}(z)}\right) d z \\
&= \nu_{n} \sqrt{\left(1-\left|\beta_{n}\right|^{2}\right)\left(1-\left|\beta_{k}\right|^{2}\right)}\left[\frac{1}{2 \mathbf{i} \pi} \oint_{\mathbb{T}} \frac{\left(z-\bar{\beta}_{n}\right) B_{n-1}^{c}(z)}{\left(z-\beta_{n}\right)\left(1-\beta_{n} z\right)\left(z-\bar{\beta}_{k}\right) B_{k-1}^{c}(z)} d z\right. \\
&=\left.\nu_{n} \sqrt{\left(1-\left|\beta_{n}\right|^{2}\right)\left(1-\left|\beta_{k}\right|^{2}\right)}\left[\frac{1}{2 \mathbf{i} \pi} \oint_{\mathbb{T}} \frac{z^{i}\left(z-\bar{\beta}_{n}\right) B_{n-1}^{c}(z) B_{k-1}(z)}{\left(z-\beta_{n}\right)\left(1-\beta_{n} z\right)\left(1-\bar{\beta}_{k} z\right)}, z=\beta_{n}\right\}\right] \\
&\left.-q \frac{\mathbf{i} 2 \Im\left\{\beta_{n}\right\} \beta_{n}^{i} B_{n-1}^{c}\left(\beta_{n}\right) B_{k-1}\left(\beta_{n}\right)}{\left(1-\beta_{n} z\right)\left(1-\bar{\beta}_{k} \beta_{n}\right)}\right]
\end{aligned}
$$

where

$$
\frac{1}{2 \mathbf{i} \pi} \oint_{\mathbb{T}} \frac{\left(z-\bar{\beta}_{n}\right) B_{n-1}^{c}(z)}{\left(z-\beta_{n}\right)\left(1-\beta_{n} z\right)\left(z-\bar{\beta}_{k}\right) B_{k-1}^{c}(z)} d z=\left\{\begin{array}{cl}
\frac{\mathbf{i} 2 \Im\left\{\beta_{n}\right\} B_{n-1}^{c}\left(\beta_{n}\right)}{\left(1-\beta_{n}^{2}\right)\left(1-\beta_{n} \beta_{k}\right) B_{k}^{c}\left(\beta_{n}\right)}, & k<n \\
\frac{1}{1-\beta_{n}^{2}}, & k=n .
\end{array}\right.
$$

This ends the proof.

Theorem 28 Let $x=J(z) \in \overline{\mathbb{C}}$ and $\alpha_{k}=J\left(\beta_{k}\right) \in \overline{\mathbb{C}}_{I}, k=1, \ldots, n$. Suppose the $n$ ORFs $\varphi_{k}, k=0, \ldots, n$, with respect to the $i$-th weight function in Table 1 are defined as before in Theorem 21, and let $\varepsilon_{n}$ and $e_{n}$ be defined as before in Theorems 24 and 26 respectively. Then the projection coefficients $\gamma_{1, k}^{[Z \varphi]}$ of $Z_{1} \varphi_{0}$ onto the basis $\left\{\varphi_{0}, \varphi_{1}\right\}$ are given by

$$
\gamma_{1,0}^{[Z \varphi]}=-\frac{\left(1-i \bmod 2-\beta_{1}\right)\left(1+\beta_{1}^{2}\right)}{2 \varepsilon_{1}} e_{1} \quad \text { and } \quad \gamma_{1,1}^{[Z \varphi]}=\frac{\left(1+\beta_{1}^{2}\right)^{2}}{2 \varepsilon_{1} \sqrt{1-\left|\beta_{1}\right|^{2}}}
$$

For $n>1$ the projection coefficients $\gamma_{n, k}^{[Z \varphi]}$ of $Z_{n} \varphi_{n-1}$ onto the basis $\left\{\varphi_{0}, \ldots, \varphi_{n}\right\}$ are given by

$$
\gamma_{n, 0}^{[Z \varphi]}=\frac{\sqrt{1-\left|\beta_{n-1}\right|^{2}}\left(1+\beta_{n}^{2}\right)^{2}}{2 \varepsilon_{n}\left(1-\beta_{n} \beta_{n-1}\right)} B_{n-2}^{c}\left(\beta_{n}\right),
$$

respectively

$$
\begin{aligned}
\gamma_{n, n}^{[Z \varphi]}=\sqrt{\frac{1-\left|\beta_{n-1}\right|^{2}}{1-\left|\beta_{n}\right|^{2}} \frac{\left(1+\beta_{n}^{2}\right)^{2}}{2\left(1-\beta_{n}^{2}\right)\left(1-\beta_{n} \beta_{n-1}\right)} \times} \\
\left\{\frac{1-\beta_{n} \beta_{n-1}}{1-\beta_{n} \bar{\beta}_{n-1}}-q \beta_{n}^{i} B_{n-2}^{c}\left(\beta_{n}\right) B_{n-1}\left(\beta_{n}\right)\right\},
\end{aligned}
$$




$$
\begin{aligned}
& \gamma_{n, n-1}^{[Z \varphi]}=\frac{\left(1+\beta_{n}^{2}\right)}{2\left(1-\beta_{n}^{2}\right)\left(1-\beta_{n} \beta_{n-1}\right)\left(1-\beta_{n} \bar{\beta}_{n-1}\right)} \times \\
&\left\{2\left[\Re\left\{\beta_{n-1}\right\}\left(1-\beta_{n}^{2}\right)+\beta_{n}\left(1-\left|\beta_{n-1}\right|^{2}\right)\right]\right. \\
&\left.\quad-q \beta_{n}^{i}\left(1-\left|\beta_{n-1}\right|^{2}\right)\left(1+\beta_{n}^{2}\right) B_{n-2}^{c}\left(\beta_{n}\right) B_{n-2}\left(\beta_{n}\right)\right\},
\end{aligned}
$$

and

$$
\begin{aligned}
\gamma_{n, k}^{[Z \varphi]} & =\frac{\sqrt{\left(1-\left|\beta_{n-1}\right|^{2}\right)\left(1-\left|\beta_{k}\right|^{2}\right)}\left(1+\beta_{n}^{2}\right)^{2}}{2\left(1-\beta_{n}^{2}\right)\left(1-\beta_{n} \beta_{n-1}\right)\left(1-\beta_{n} \bar{\beta}_{k}\right)} \times \\
& \frac{B_{n-2}^{c}\left(\beta_{n}\right)}{B_{k}^{c}\left(\beta_{n}\right)}\left\{\frac{1-\beta_{n} \bar{\beta}_{k}}{1-\beta_{n} \beta_{k}}-q \beta_{n}^{i} B_{k}^{c}\left(\beta_{n}\right) B_{k-1}\left(\beta_{n}\right)\right\}, \quad k=1, \ldots, n-2 .
\end{aligned}
$$

Proof. First, note that for $n=1$ and $k \in\{0,1\}$ it holds that

$$
\frac{1}{E_{1}}\left\langle\varphi_{1}, \varphi_{k}\right\rangle_{\mathfrak{F}}=\left[1+D_{1} / Z_{0}\left(\alpha_{1}\right)\right]\left\langle Z_{1} \varphi_{0}, \varphi_{k}\right\rangle_{\mathfrak{F}}+D_{1}\left\langle\varphi_{0}, \varphi_{k}\right\rangle_{\mathfrak{F}}
$$

where $E_{1}$ and $D_{1}$ are given by (35) and (36) respectively. Some computations now yields the expressions for $n=1$.

Next, note that it follows from Theorem 26 and the expressions for $\gamma_{n, k}^{[Q]}$ and $\gamma_{n, k}^{[P]}$ that $\gamma_{n, k}^{\left[\varphi^{*}\right]}$ and $\gamma_{n, k}^{[Z \varphi]}$ are related by

$$
\begin{aligned}
\bar{\nu}_{n} \gamma_{n, k}^{\left[\varphi^{*}\right]} & -\frac{1-\bar{\beta}_{n} \bar{\beta}_{n-1}}{1-\beta_{n} \bar{\beta}_{n-1}} \delta_{n, k} \\
& =\sqrt{\frac{1-\left|\beta_{n}\right|^{2}}{1-\left|\beta_{n-1}\right|^{2}}} \frac{\mathbf{i} 2 \Im\left\{\beta_{n}\right\}\left(1+\bar{\beta}_{n-1}^{2}\right)}{\left(1-\beta_{n} \bar{\beta}_{n-1}\right)\left(1+\beta_{n}^{2}\right)} e_{n}\left(\delta_{n-1, k}+\frac{\gamma_{n, k}^{[Z \varphi]}}{Z_{n-1}^{c}\left(\alpha_{n}\right)}\right),
\end{aligned}
$$

for $k=0, \ldots, n$. It is easily verified that these equalities are satisfied for $n=1$. For $n>1$ and $k=0, \ldots, n-2$ the equality is satisfied iff

$$
\gamma_{n, k}^{[Z \varphi]}=\frac{\bar{\nu}_{n} \gamma_{n, k}^{\left[\varphi^{*}\right]}}{1 / Z_{n-1}^{c}\left(\alpha_{n}\right)} \sqrt{\frac{1-\left|\beta_{n-1}\right|^{2}}{1-\left|\beta_{n}\right|^{2}}} \frac{\left(1-\beta_{n} \bar{\beta}_{n-1}\right)\left(1+\beta_{n}^{2}\right)}{\mathbf{i} 2 \Im\left\{\beta_{n}\right\}\left(1+\bar{\beta}_{n-1}^{2}\right)} .
$$

For $k=0$ this yields

$$
\begin{aligned}
\gamma_{n, 0}^{[Z \varphi]} & =\frac{\zeta_{n-1}^{c}\left(\beta_{n}\right)\left(1-\beta_{n} \bar{\beta}_{n-1}\right)}{\left(1+\bar{\beta}_{n-1}^{2}\right) / Z_{n-1}^{c}\left(\alpha_{n}\right)} \cdot \frac{\sqrt{1-\left|\beta_{n-1}\right|^{2}}}{\varepsilon_{n}}\left(1+\beta_{n}^{2}\right) B_{n-2}^{c}\left(\beta_{n}\right) \\
& =\frac{\sqrt{1-\left|\beta_{n-1}\right|^{2}}\left(1+\beta_{n}^{2}\right)^{2}}{2 \varepsilon_{n}\left(1-\beta_{n} \beta_{n-1}\right)} B_{n-2}^{c}\left(\beta_{n}\right),
\end{aligned}
$$


while for $0<k \leqslant n-2$ we get that

$$
\begin{aligned}
& \gamma_{n, k}^{[Z \varphi]}= \frac{\zeta_{n-1}^{c}\left(\beta_{n}\right)\left(1-\beta_{n} \bar{\beta}_{n-1}\right)}{\left(1+\bar{\beta}_{n-1}^{2}\right) / Z_{n-1}^{c}\left(\alpha_{n}\right)} \cdot \frac{\sqrt{\left(1-\left|\beta_{n-1}\right|^{2}\right)\left(1-\left|\beta_{k}\right|^{2}\right)}\left(1+\beta_{n}^{2}\right)}{\left(1-\beta_{n}^{2}\right)\left(1-\beta_{n} \bar{\beta}_{k}\right)} \times \\
& \frac{B_{n-2}^{c}\left(\beta_{n}\right)}{B_{k}^{c}\left(\beta_{n}\right)}\left\{\frac{1-\beta_{n} \bar{\beta}_{k}}{1-\beta_{n} \beta_{k}}-q \beta_{n}^{i} B_{k}^{c}\left(\beta_{n}\right) B_{k-1}\left(\beta_{n}\right)\right\} \\
&=\frac{\sqrt{\left(1-\left|\beta_{n-1}\right|^{2}\right)\left(1-\left|\beta_{k}\right|^{2}\right)}\left(1+\beta_{n}^{2}\right)^{2}}{2\left(1-\beta_{n}^{2}\right)\left(1-\beta_{n} \beta_{n-1}\right)\left(1-\beta_{n} \bar{\beta}_{k}\right)} \times \\
& \frac{B_{n-2}^{c}\left(\beta_{n}\right)}{B_{k}^{c}\left(\beta_{n}\right)}\left\{\frac{1-\beta_{n} \bar{\beta}_{k}}{1-\beta_{n} \beta_{k}}-q \beta_{n}^{i} B_{k}^{c}\left(\beta_{n}\right) B_{k-1}\left(\beta_{n}\right)\right\} .
\end{aligned}
$$

So, it remains to prove the expressions for $k \in\{n-1, n\}$. For $k=n$ it holds that

$$
\begin{aligned}
\bar{\nu}_{n} \gamma_{n, n}^{\left[\varphi^{*}\right]}- & \frac{1-\bar{\beta}_{n} \bar{\beta}_{n-1}}{1-\beta_{n} \bar{\beta}_{n-1}} \\
& =\frac{\mathrm{i} 2 \Im\left\{\beta_{n}\right\} \zeta_{n-1}^{c}\left(\beta_{n}\right)}{1-\beta_{n}^{2}}\left\{\frac{1-\beta_{n} \beta_{n-1}}{1-\beta_{n} \bar{\beta}_{n-1}}-q \beta_{n}^{i} B_{n-2}^{c}\left(\beta_{n}\right) B_{n-1}\left(\beta_{n}\right)\right\},
\end{aligned}
$$

so that

$$
\begin{aligned}
& \gamma_{n, n}^{[Z \varphi]}= \frac{\zeta_{n-1}^{c}\left(\beta_{n}\right)\left(1-\beta_{n} \bar{\beta}_{n-1}\right)}{\left(1+\bar{\beta}_{n-1}^{2}\right) / Z_{n-1}^{c}\left(\alpha_{n}\right)} \cdot \sqrt{\frac{1-\left|\beta_{n-1}\right|^{2}}{1-\left|\beta_{n}\right|^{2}}}\left(\frac{1+\beta_{n}^{2}}{1-\beta_{n}^{2}}\right) \times \\
&\left\{\frac{1-\beta_{n} \beta_{n-1}}{1-\beta_{n} \bar{\beta}_{n-1}}-q \beta_{n}^{i} B_{n-2}^{c}\left(\beta_{n}\right) B_{n-1}\left(\beta_{n}\right)\right\} \\
& \sqrt{\frac{1-\left|\beta_{n-1}\right|^{2}}{1-\left|\beta_{n}\right|^{2}}} \frac{\left(1+\beta_{n}^{2}\right)^{2}}{2\left(1-\beta_{n}^{2}\right)\left(1-\beta_{n} \beta_{n-1}\right)} \times \\
&\left\{\frac{1-\beta_{n} \beta_{n-1}}{1-\beta_{n} \bar{\beta}_{n-1}}-q \beta_{n}^{i} B_{n-2}^{c}\left(\beta_{n}\right) B_{n-1}\left(\beta_{n}\right)\right\} .
\end{aligned}
$$

Finally, for $k=n-1$ we have that

$$
\frac{\gamma_{n, n-1}^{[Z \varphi]}}{Z_{n-1}^{c}\left(\alpha_{n}\right)}=\frac{\left(1-\left|\beta_{n-1}\right|^{2}\right)\left(1+\beta_{n}^{2}\right)}{\left(1+\bar{\beta}_{n-1}^{2}\right)\left(1-\beta_{n}^{2}\right)}\left\{\frac{1-\beta_{n} \bar{\beta}_{n-1}}{1-\beta_{n} \beta_{n-1}}-q \beta_{n}^{i} B_{n-1}^{c}\left(\beta_{n}\right) B_{n-2}\left(\beta_{n}\right)\right\}-1,
$$

where it holds that

$$
\begin{aligned}
\left(1-\left|\beta_{n-1}\right|^{2}\right)\left(1+\beta_{n}^{2}\right)\left(1-\beta_{n} \bar{\beta}_{n-1}\right)-\left(1+\bar{\beta}_{n-1}^{2}\right)\left(1-\beta_{n}^{2}\right)\left(1-\beta_{n} \beta_{n-1}\right) & \\
= & 2\left(\beta_{n}-\bar{\beta}_{n-1}\right)\left[\Re\left\{\beta_{n-1}\right\}\left(1-\beta_{n}^{2}\right)+\beta_{n}\left(1-\left|\beta_{n-1}\right|^{2}\right)\right]
\end{aligned}
$$


so that

$$
\begin{array}{r}
\frac{\gamma_{n, n-1}^{[Z \varphi]}}{Z_{n-1}^{c}\left(\alpha_{n}\right)}=\frac{\zeta_{n-1}^{c}\left(\beta_{n}\right)}{\left(1+\bar{\beta}_{n-1}^{2}\right)\left(1-\beta_{n}^{2}\right)} \times\left\{2\left[\Re\left\{\beta_{n-1}\right\}\left(1-\beta_{n}^{2}\right)+\beta_{n}\left(1-\left|\beta_{n-1}\right|^{2}\right)\right]\right. \\
\left.-q \beta_{n}^{i}\left(1-\left|\beta_{n-1}\right|^{2}\right)\left(1+\beta_{n}^{2}\right) B_{n-2}^{c}\left(\beta_{n}\right) B_{n-2}\left(\beta_{n}\right)\right\} .
\end{array}
$$

The expression for $k=n-1$ now easily follows.

\section{Numerical experiments}

For the numerical experiments that follow, we consider the sequence of poles

$$
\alpha_{k}=\frac{(-1)^{k} 3 \mathbf{i}}{4}, \text { and hence, } \beta_{k}=\frac{(-1)^{k+1} \mathbf{i}}{2}, \quad k=1, \ldots, n .
$$

The recurrence coefficients $a_{k-1}, b_{k-1}$ and $c_{k-1}$, for $k=1, \ldots, n$, are then given by

$$
\begin{gathered}
c_{k-1}=E_{k}^{-1}= \begin{cases}\frac{e_{1} \sqrt{3}}{4}, & k=1 \\
\frac{1}{2}, & k>1,\end{cases} \\
b_{k-1}=-D_{k}= \begin{cases}-\frac{e_{1}^{2}}{2}\left(1-i \bmod 2-\frac{\mathbf{i}}{2}\right), & k=1 \\
-\frac{\mathbf{i}}{4}, & k=2 \\
0, & k>2\end{cases}
\end{gathered}
$$

and

$$
a_{k-1}=C_{k}= \begin{cases}\frac{e_{1} 5 \sqrt{3}}{12}, & k=2 \\ \frac{1}{2}, & k>2\end{cases}
$$

where $e_{1}=\sqrt{2}$ if $i=1$, and $e_{1}=1$ otherwise.

Let the Chebyshev pORF $P_{n}$ be given by $P_{n}(x)=5\left\{\varphi_{n}(x)+\tau_{n} \varphi_{n}^{*}(x)\right\}$, with $n>0$ and $\tau_{n} \in \mathbb{C}$. From Theorem 24 it follows that $P_{n} \in{ }^{r} \mathcal{L}_{n}$ if

$$
\tau_{1} \neq-1 \quad \text { and } \quad \tau_{1} \neq \begin{cases}-5, & i=1 \\ -\frac{1}{2}(3-\mathbf{i}), & i=2 \\ -2, & i=3\end{cases}
$$

respectively

$$
\tau_{n} \notin\left\{-1,-\frac{5}{3},-\frac{3}{5}\right\}, \quad n>1 .
$$

Further, the zeros of $P_{n}(x)$ are all real and simple for $\tau_{n} \in \mathbb{T} \backslash\{-1\}$. In order to have them all in $I$, it follows from Theorem 25 that $\tau_{n}$ is bounded by the condition that

$$
\tilde{\beta}_{n}=\frac{(-1)^{n-1} \mathbf{i}}{2}\left(\frac{1-\tau_{n}}{1+\tau_{n}}\right) \in\left\{\begin{array}{cl}
{[-1,1],} & i=1 \\
{\left[-1, \frac{3(2 n+3)}{6 n-1}\right],} & i=2 \\
{\left[-\frac{3 n+7}{3 n+2}, \frac{3 n+7}{3 n+2}\right],} & i=3
\end{array}\right.
$$


From Theorem 26 we deduce that the corresponding Chebyshev qORF is given by

$$
Q_{n}(x)= \begin{cases}5\left(1+\tau_{1}\right) \varphi_{1}(x)+\frac{10 \sqrt{3} \tau_{1} \mathbf{i}}{3} e_{1} \frac{Z_{1}(x)}{Z_{0}(x)} \varphi_{0}, & n=1 \\ \left(5+3 \tau_{n}\right) \varphi_{n}(x)-(-1)^{n} 4 \tau_{n} \mathbf{i} \varphi_{n-1}(x), & n>1\end{cases}
$$

Let us first consider the case in which $n>2$. From (46) it directly follows that the projection coefficients $\gamma_{n, k}^{[P]}=\gamma_{n, k}^{[Q]}$ are given by

$$
\gamma_{n, k}^{[Q]}= \begin{cases}0, & k=0, \ldots, n-2 \\ (-1)^{n+1} 4 \tau_{n} \mathbf{i}, & k=n-1 \\ 5+3 \tau_{n} & k=n,\end{cases}
$$

while it follows from (19)-(20) that the recurrence coefficients $a_{n-1}^{[P]}=a_{n-1}^{[Q]}$, $b_{n-1}^{[P]}=b_{n-1}^{[Q]}$ and $c_{n-1}^{[P]}=c_{n-1}^{[Q]}$ are given by

$$
c_{n-1}^{[Q]}=\left(E_{n}^{[Q]}\right)^{-1}=\frac{3}{10\left(3+5 \tau_{n}\right)}, \quad b_{n-1}^{[Q]}=-D_{n}^{[Q]}=\frac{(-1)^{n} 6 \tau_{n} \mathbf{i}}{5\left(3+5 \tau_{n}\right)}
$$

and

$$
a_{n-1}^{[Q]}=C_{n}^{[Q]}=\frac{3\left(5+3 \tau_{n}\right)}{10\left(3+5 \tau_{n}\right)} .
$$

Note that $C_{n}^{[Q]}$ and $D_{n}^{[Q]}$ satisfy the relation given by (16), and that the expression for $D_{n}^{[Q]}$ could also be obtained by means of (43).

Let $D_{n}^{[Q]}=x+\mathbf{i} y$. Taking into account that $\left|\alpha_{n-1}\right|^{2}=\left[\Im\left\{\alpha_{n-1}\right\}\right]^{2}=1 / 4$, it follows that $v_{n}$, defined as before in Theorem 7 , equals zero iff

$$
x^{2}+\left(y+(-1)^{n} \frac{3}{8}\right)^{2}=\left(\frac{9}{40}\right)^{2} .
$$

In other words, $D_{n}^{[Q]}$ should lie on a circle in the complex plain with center $\left(0,(-1)^{n+1} \frac{3}{8}\right)$ and radius $\frac{9}{40}$. Figure 1 graphically shows the circle (49) for $n$ odd $^{4}$. Also the graph of $D_{n}^{[Q]}$, given by (48) and (43), is plotted as a function of $\tau_{n}$ and $\tilde{\beta}_{n}$, for $\tilde{\beta}_{n} \in I$. The values of $D_{n}^{[Q]}$ for $\tilde{\beta}_{3} \in\{27 / 17,16 / 11,-16 / 11\}$ (i.e., the endpoints of the intervals (45) for $i=2$ and $i=3$, with $n=3$ ) and for $\tilde{\beta}_{n}=\infty$ are marked too. Clearly, the graph of $D_{n}^{[Q]}$ coincides with the circle (49) for $\tau_{n} \in \mathbb{T}$ or $\tilde{\beta}_{n} \in \overline{\mathbb{R}}$.

Next, for $n=2$, the expressions (47) for the projection coefficients $\gamma_{n, k}^{[Q]}$ remain valid, but the recurrence coefficients are now given by

$$
c_{1}^{[Q]}=\left(E_{2}^{[Q]}\right)^{-1}=\frac{3}{10\left(3+5 \tau_{2}\right)}, \quad b_{1}^{[Q]}=-D_{2}^{[Q]}=\frac{3\left(\tau_{2}-1\right) \mathbf{i}}{4\left(3+5 \tau_{2}\right)}
$$

and

$$
a_{1}^{[Q]}=C_{2}^{[Q]}=\frac{e_{1}\left(5+3 \tau_{2}\right) \sqrt{3}}{4\left(3+5 \tau_{2}\right)} .
$$

\footnotetext{
4 The result for $n$ even is similar.
} 


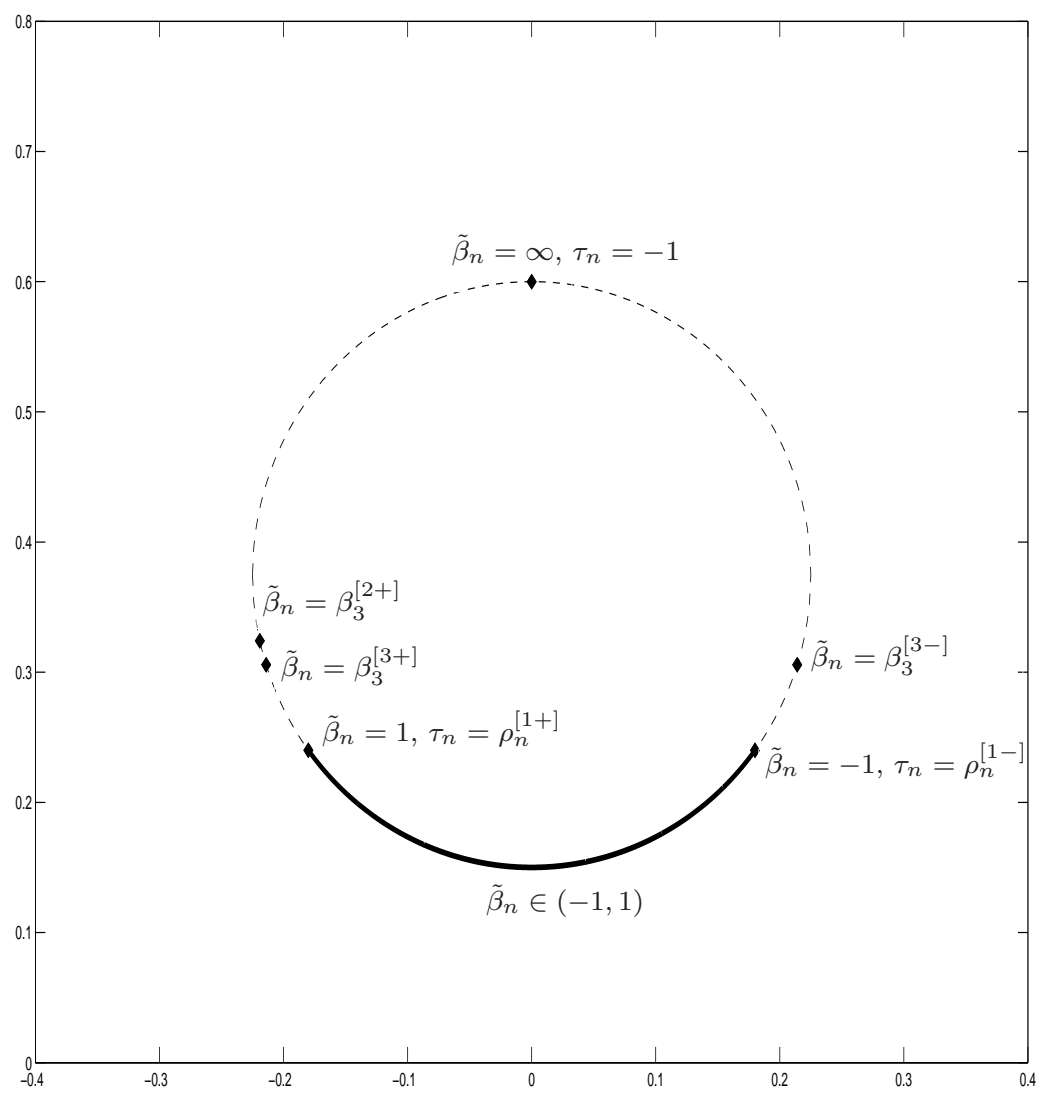

Fig. 1 The circle (49), with $n=$ odd, together with the graph of $D_{n}^{[Q]}$, given by (48) and (43), as a function of $\tau_{n} \in \mathbb{T}$ and $\tilde{\beta}_{n} \in \overline{\mathbb{R}}$.

For $n=1$, on the other hand, we find with the aid of Theorem 27 or 28 that the projection coefficients are given by

$$
\gamma_{1,0}^{[Q]}=\frac{5 \sqrt{3} \tau_{1} \mathbf{i}}{2 \varepsilon_{1}}, \quad \varepsilon_{1}=\left\{\begin{aligned}
\frac{5 \sqrt{2}}{8}, & i=1 \\
\frac{2+\mathbf{i}}{\mathbf{i}} & i=2 \\
1, & i=3
\end{aligned}\right.
$$

and

$$
\gamma_{1,1}^{[Q]}=\left\{\begin{array}{lr}
5+\tau_{1}, & i=1 \\
5+(3+\mathbf{i}) \tau_{1} & i=2 \\
5+\frac{5 \tau_{1}}{2}, & i=3
\end{array}\right.
$$


while the recurrence coefficients are given by

$$
\begin{aligned}
& c_{0}^{[Q]}=\left(E_{1}^{[Q]}\right)^{-1}=\frac{e_{1} \sqrt{3}}{20\left(1+\tau_{1}\right)} \text { and } \\
& \qquad b_{0}^{[Q]}=-D_{1}^{[Q]}=-\frac{e_{1}^{2}}{2}\left[1-i \bmod 2-\left(\frac{1-\tau_{1}}{1+\tau_{1}}\right) \frac{\mathbf{i}}{2}\right] .
\end{aligned}
$$

Finally, in the examples that follow the computations were done with MAT$\mathrm{LAB}^{\circledR} 7^{5}$.

Example 29 For the first example we consider the case in which $\tau_{n}=1$. We then computed the zeros of $P_{n}$, and the corresponding weights in the rational Gauss-Chebyshev quadrature formula, by means of the GEP (10) ('EP') and the GEP (21) ('GEP'). Let us denote the results by $x^{[\mathrm{EP}]}:=\left\{x_{n, j}^{[\mathrm{EP}]}\right\}_{j=1}^{n}$ and $\lambda^{[\mathrm{EP}]}:=\left\{\lambda_{n, j}^{[\mathrm{EP}]}\right\}_{j=1}^{n}$ for the GEP (10), respectively $x^{[\mathrm{GEP}]}:=\left\{x_{n, j}^{[\mathrm{GEP}]}\right\}_{j=1}^{n}$ and $\lambda^{[\mathrm{GEP}]}:=\left\{\lambda_{n, j}^{[\mathrm{GEP}]}\right\}_{j=1}^{n}$ for the GEP (21)). Note that in this case, the zeros of $P_{n}$ are also zeros of a Chebyshev nORF $\varphi_{n}^{[\infty]} \in \mathcal{L}_{n}^{[\infty]}$. In [40] a fast and efficient algorithm ('CHEB') has been presented to compute the nodes and weights in rational Gauss-Chebyshev quadrature formulas. So, we used this algorithm too to compute the zeros of $\varphi_{n}^{[\infty]}$, and the corresponding weights in the rational Gauss-Chebyshev quadrature formula (let us denote the results by $\left\{x_{n, j}^{[\mathrm{CHEB}]}\right\}_{j=1}^{n}$ and $\left\{\lambda_{n, j}^{[\mathrm{CHEB}]}\right\}_{j=1}^{n}$. In Tables $2-4$ the maximal relative difference ${ }^{6}$

$$
\begin{aligned}
& \Delta_{n}(x)=\max _{1 \leqslant j \leqslant n}\left|\frac{x_{n, j}-x_{n, j}^{[\mathrm{CHEB}]}}{x_{n, j}^{[\mathrm{CHEB}]}}\right| \\
& \text { and } \Delta_{n}(\lambda)=\max _{1 \leqslant j \leqslant n}\left|\frac{\lambda_{n, j}-\lambda_{n, j}^{[\mathrm{CHEB}]}}{\lambda_{n, j}^{[\mathrm{CHEB}]}}\right|, \quad n=25 k, k=1, \ldots, 10,
\end{aligned}
$$

is given for the three Chebyshev weight functions in Table 1.

In general, 'GEP' will be more efficient and accurate compared with 'EP', because the former only requires the computation of two coefficients, while the latter requires the computation of $(n+1)$ projection coefficients by means of inner products (for which explicit expressions not always exist, so that they may need to be approximated numerically). Of course, due to the explicit expressions derived above, and the specific sequence of poles, this cannot be deduced from the tables. In the case of a functional defined by the Chebyshev weight function, however, most of the computational cost goes into solving the generalized eigenvalue problem for $n$ sufficiently large, so that the efficiency of both methods is more or less the same. In this case, due to the explicit

\footnotetext{
5 Matlab is a registered trademark of The MathWorks, Inc.

6 The weights $\lambda_{n, j}$ are always strictly positive, so that the relative difference can always be considered. The zeros $x_{n, j}$, however, are not bounded away from 0 . So, whenever $\varphi_{n}^{[\infty]}$ appeared to have a zero in $x=0$, the absolute difference was considered instead in this zero.
} 
Table 2 Maximal relative difference (50) of the computed nodes and weights for $i=1$.

\begin{tabular}{|c||c|c||c|c|}
\hline$n$ & $\Delta_{n}\left(x^{\mathrm{EP}]}\right)$ & $\Delta_{n}\left(\lambda^{[\mathrm{EP}]}\right)$ & $\Delta_{n}\left(x^{[\mathrm{GEP}]}\right)$ & $\Delta_{n}\left(\lambda^{[\mathrm{GEP}]}\right)$ \\
\hline 25 & $3.210^{-15}$ & $4.110^{-14}$ & $3.410^{-15}$ & $4.510^{-14}$ \\
50 & $1.310^{-14}$ & $3.010^{-13}$ & $1.110^{-14}$ & $1.010^{-13}$ \\
75 & $6.810^{-15}$ & $1.010^{-12}$ & $4.610^{-15}$ & $2.310^{-13}$ \\
100 & $3.010^{-14}$ & $9.110^{-13}$ & $2.710^{-14}$ & $1.110^{-12}$ \\
125 & $2.510^{-14}$ & $1.510^{-12}$ & $2.610^{-14}$ & $9.710^{-13}$ \\
150 & $6.510^{-14}$ & $3.410^{-12}$ & $7.110^{-14}$ & $2.210^{-12}$ \\
175 & $2.910^{-14}$ & $3.010^{-12}$ & $2.310^{-14}$ & $3.510^{-12}$ \\
200 & $4.910^{-14}$ & $9.110^{-12}$ & $4.610^{-14}$ & $6.910^{-12}$ \\
225 & $3.010^{-14}$ & $7.610^{-12}$ & $3.310^{-14}$ & $6.110^{-12}$ \\
250 & $5.910^{-14}$ & $9.310^{-12}$ & $5.610^{-14}$ & $2.610^{-11}$ \\
\hline
\end{tabular}

Table 3 Maximal relative difference (50) of the computed nodes and weights for $i=2$.

\begin{tabular}{|c||c|c||c|c|}
\hline$n$ & $\Delta_{n}\left(x^{\mathrm{EP}}\right)$ & $\Delta_{n}\left(\lambda^{\mathrm{EP}}\right)$ & $\Delta_{n}\left(x^{\mathrm{GEP}}\right)$ & $\Delta_{n}\left(\lambda^{\mathrm{GEP}}\right)$ \\
\hline 25 & $4.310^{-15}$ & $6.210^{-14}$ & $2.810^{-15}$ & $4.110^{-14}$ \\
50 & $1.810^{-14}$ & $4.010^{-13}$ & $1.610^{-14}$ & $2.010^{-13}$ \\
75 & $9.510^{-15}$ & $9.310^{-13}$ & $1.110^{-14}$ & $3.210^{-13}$ \\
100 & $3.010^{-14}$ & $4.610^{-12}$ & $3.010^{-14}$ & $3.710^{-13}$ \\
125 & $8.710^{-14}$ & $2.610^{-12}$ & $6.410^{-14}$ & $5.810^{-12}$ \\
150 & $2.610^{-14}$ & $3.110^{-12}$ & $2.910^{-14}$ & $4.710^{-12}$ \\
175 & $1.010^{-13}$ & $4.910^{-12}$ & $1.110^{-13}$ & $1.510^{-12}$ \\
200 & $1.710^{-13}$ & $1.110^{-11}$ & $2.010^{-13}$ & $8.310^{-12}$ \\
225 & $4.910^{-14}$ & $1.210^{-11}$ & $4.410^{-14}$ & $9.110^{-12}$ \\
250 & $6.610^{-14}$ & $1.310^{-11}$ & $7.310^{-14}$ & $1.110^{-11}$ \\
\hline
\end{tabular}

expressions derived above, we may assume that both methods are as accurate too (as indicated by the tables). ${ }^{7}$

Compared with 'CHEB', the tables clearly show that 'EP' and 'GEP' are more or less as accurate for small $n$, but that they become less accurate for increasing $n$. We should also point out that the more the poles approach the interval, the less accurate the results. On the other hand, 'CHEB' can only be used if $\tilde{\beta}_{n}$, given by (42), is strictly less than one in absolute value. Finally, 'CHEB' computes the nodes and weights in $\mathcal{O}(n \cdot m)$ flops, where $m$ represents the number of different poles; see [17]. For this reason, 'CHEB' is theoretically more efficient than 'EP' and 'GEP'. However, this order is only reached for larger $n$, while in practical applications, quadrature rules that converge fast such that an accurate result up to machine precision can be obtained for $n$ as small as possible, are the most interesting. For small $n$, the efficiency of 'EP' and 'GEP' is comparable with the efficiency of 'CHEB', because their implementation is much shorter and more straightforward. If, however, the quadrature rule converges too slowly, convergence may perhaps be sped up by considering another sequence of poles.

\footnotetext{
7 In the tables we took 'CHEB' as reference to investigate the accuracy of 'EP' and 'GEP'. When using the computed nodes and weights for numerical integration, the better results were always obtained with the aid 'CHEB', so that we indeed may assume that the nodes and weights computed by means of 'CHEB' are the most accurate, and hence, a good reference.
} 
Table 4 Maximal relative difference (50) of the computed nodes and weights for $i=3$.

\begin{tabular}{|c||c|c||c|c|}
\hline$n$ & $\Delta_{n}\left(x^{\mathrm{EP}}\right)$ & $\Delta_{n}\left(\lambda^{\mathrm{EP}}\right)$ & $\Delta_{n}\left(x^{\mathrm{GEP}}\right)$ & $\Delta_{n}\left(\lambda^{\mathrm{GEP}}\right)$ \\
\hline 25 & $3.910^{-15}$ & $2.210^{-14}$ & $4.210^{-15}$ & $2.510^{-14}$ \\
50 & $1.610^{-14}$ & $3.010^{-13}$ & $1.510^{-14}$ & $4.310^{-13}$ \\
75 & $1.110^{-14}$ & $1.210^{-12}$ & $1.210^{-14}$ & $1.310^{-12}$ \\
100 & $5.110^{-14}$ & $1.810^{-12}$ & $4.310^{-14}$ & $7.410^{-13}$ \\
125 & $2.410^{-14}$ & $4.010^{-12}$ & $2.410^{-14}$ & $2.210^{-12}$ \\
150 & $6.610^{-14}$ & $3.410^{-12}$ & $6.210^{-14}$ & $4.310^{-12}$ \\
175 & $2.510^{-14}$ & $6.610^{-12}$ & $2.610^{-14}$ & $3.710^{-12}$ \\
200 & $1.310^{-13}$ & $8.610^{-12}$ & $1.410^{-13}$ & $6.210^{-12}$ \\
225 & $3.910^{-14}$ & $5.010^{-12}$ & $4.810^{-14}$ & $5.310^{-12}$ \\
250 & $1.110^{-13}$ & $3.010^{-12}$ & $1.210^{-13}$ & $1.310^{-11}$ \\
\hline
\end{tabular}

Example 30 For the last example we consider the integral

$$
\mathfrak{F}\{f\}=\int_{I} f(x) \sqrt{1-x^{2}} d x,
$$

where the function

$$
f(x)=\sin \left(\frac{1}{x^{2}+(3 / 4)^{2}}\right)
$$

is taken from [18, Ex. 5.3]. This function has an essential singularity in $x=\frac{3 \mathbf{i}}{4}$ and $x=-\frac{3 \mathbf{i}}{4}$. Since an essential singularity can be viewed as a pole of infinite multiplicity, this suggests taking

$$
\alpha_{k}=\frac{(-1)^{k} 3 \mathbf{i}}{4}, \quad k=1, \ldots, n .
$$

We calculated the exact solution of $\mathfrak{F}\{f\}$ in multiprecision with MAPLE ${ }^{\circledR} 10^{8}$. Table 5 then gives the relative error on the approximation of (51) by means of an $n$-point rational Gauss-Chebyshev quadrature formula based on the zeros of the Chebyshev pORF $P_{n}(x)=5\left[\varphi_{n}(x)+\tau_{n} \varphi_{n}^{*}(x)\right], n=3, \ldots, 12$, where $\tau_{n}$ is chosen in such a way that $\tilde{\beta}_{n}$, given by (45), is equal to either 0 or $\frac{3 n+7}{3 n+2}$. We used the GEP (21) to compute the nodes and weight.

Finally, note that $P_{n}(x)$ should have a zero in $x=1$ whenever $\tilde{\beta}_{n}=\frac{3 n+7}{3 n+2}$. To verify this, the largest and smallest zero of $P_{n}(x)$ is given in Table 6 .

\section{Conclusion}

We provided a generalized eigenvalue problem to compute the zeros of a so-called quasi-orthogonal rational function (qORF) and the corresponding weights in the rational Gaussian quadrature formula. In contrast with orthogonal rational functions (ORFs) on a subset of the real line with poles among $\left\{\alpha_{1}, \ldots, \alpha_{n}\right\}$, the zeros of a qORF depended on a parameter $\tau \in$ $(\mathbb{C} \cup\{\infty\})$, which could always be chosen in such a way that the zeros are

\footnotetext{
8 MAPLE is a registered trademark of Waterloo Maple, Inc.
} 
Table 5 Relative error on the estimation of (51).

\begin{tabular}{|c|c|c|}
\hline$n$ & $\beta_{n}=0$ & $\beta_{n}=\frac{3 n+7}{3 n+2}$ \\
\hline 3 & $1.710^{-3}$ & $1.610^{-3}$ \\
4 & $4.110^{-4}$ & $2.310^{-4}$ \\
5 & $7.610^{-6}$ & $4.210^{-6}$ \\
6 & $1.110^{-6}$ & $4.410^{-7}$ \\
7 & $1.510^{-8}$ & $5.910^{-9}$ \\
8 & $1.610^{-9}$ & $4.910^{-10}$ \\
9 & $1.710^{-11}$ & $5.210^{-12}$ \\
10 & $1.410^{-12}$ & $3.610^{-13}$ \\
11 & $1.310^{-14}$ & $4.210^{-15}$ \\
12 & $9.310^{-16}$ & $7.710^{-16}$ \\
\hline
\end{tabular}

Table 6 The largest and smallest zero of $P_{n}(x)=5\left[\varphi_{n}(x)+\tau_{n} \varphi_{n}^{*}(x)\right]$ for $\tau_{n}$ chosen in such a way that $\tilde{\beta}_{n}=\frac{3 n+7}{3 n+2}$, where $\tilde{\beta}_{n}$ is given by $(45)$.

\begin{tabular}{|c|c|c|}
\hline$n$ & largest zero $\left(\right.$ real part $^{a}$ ) & smallest zero $\left(\right.$ real part $^{a}$ ) \\
\hline 3 & 1.000000000000000 & -0.4840240577449624 \\
4 & 1.000000000000000 & -0.6354870802328475 \\
5 & 0.9999999999999993 & -0.7277087451950761 \\
6 & 1.000000000000000 & -0.7887830761797099 \\
7 & 0.9999999999999997 & -0.8314675794018541 \\
8 & 0.9999999999999998 & -0.8624940438879204 \\
9 & 0.9999999999999996 & -0.8857482440642103 \\
10 & 1.000000000000000 & -0.9036166621909483 \\
11 & 0.9999999999999993 & -0.9176350836535240 \\
12 & 1.000000000000001 & -0.9288292785300265 \\
\hline
\end{tabular}

a The imaginary parts are zero up to machine precision.

all real and simple. First, we showed that the zeros of a qORF are - under some mild conditions on the parameter $\tau$ - zeros of an ORF with poles among $\left\{\alpha_{1}, \ldots, \alpha_{n-1}, \alpha\right\}$, where $\alpha$ depended on the parameter $\tau$. Then, we gave a relation between qORFs and the so-called para-orthogonal rational functions. Next, a condition was given for the parameter $\tau$ so that the zeros of a qORF are all real and simple. Finally, some illustrative and numerical examples were given for the case in which orthogonality was with respect to the Chebyshev weight functions $(1-x)^{a}(1+x)^{b}$ on the interval $[-1,1]$, where $a, b \in\left\{ \pm \frac{1}{2}\right\}$.

\section{References}

1. R. Baltensperger, J.-P. Berrut, and Y. Dubey. "The linear rational pseudospectral method with preassigned poles", Numerical Algorithms 33(1-4):53-63, 2003. International Conference on Numerical Algorithms, Vol. I (Marrakesh, 2001).

2. J.-P. Berrut and R. Baltensperger. "The linear rational pseudospectral method for boundary value problems", BIT 41 (5, suppl.):868-879, 2001. BIT 40th Anniversary Meeting.

3. J.-P. Berrut and H.D. Mittelmann. "Optimized point shifts and poles in the linear rational pseudospectral method for boundary value problems", Journal of Computational Physics 204(1):292-301, 2005. 
4. A. Bultheel and M.J. Cantero. "A matrical computation of rational quadrature formulas on the unit circle", Numerical Algorithms 52(1):47-68, 2009.

5. A. Bultheel, R. Cruz-Barroso, K. Deckers, and P. González-Vera. "Rational Szegö quadratures associated with Chebyshev weight functions", Mathematics of Computation 78(266):1031-1059, 2009.

6. A. Bultheel, P. González Vera, E. Hendriksen, and O. Njåstad. Orthogonal Rational Functions, volume 5 of Cambridge Monographs on Applied and Computational Mathematics, Cambridge University Press, Cambridge, 1999.

7. A. Bultheel, P. González-Vera, E. Hendriksen, and O. Njåstad. "Orthogonal rational functions and quadrature on the real half line", Journal of Complexity 19(3):212-230, 2003.

8. A. Bultheel, P. González-Vera, E. Hendriksen, and O. Njåstad. "Orthogonal rational functions on the real half line with poles in $[-\infty, 0]$ ", Journal of Computational and Applied Mathematics 179(1-2):121-155, 2005.

9. A. Bultheel, P. González-Vera, E. Hendriksen, and O. Njåstad. "Quadrature and orthogonal rational functions", Journal of Computational and Applied Mathematics 127(12):67-91, 2001. Invited paper.

10. M.J. Cantero, L. Moral, and L. Velázquez. "Measures and paraorthogonal polynomials on the unit circle", East Journal on Approximations 8(4):447-464, 2002.

11. T.S. Chihara. "On quasi-orthogonal polynomials", Proceedings of the American Mathematical Society 8:765-767, 1957.

12. D. Day and L. Romero. "Roots of polynomials expressed in terms of orthogonal polynomials", SIAM Journal on Numerical Analysis 43(5):1969-1987 (electronic), 2005.

13. K. Deckers. Orthogonal Rational Functions: Quadrature, Recurrence and Rational Krylov, Phd thesis, Department of Computer Science, Katholieke Universiteit Leuven, Leuven, Belgium, February 2009. 270 + lxiv pages.

14. K. Deckers and A. Bultheel. "Orthogonal rational functions with complex poles: The Favard theorem", Journal of Mathematical Analysis and Applications 356(2):764-768, 2009.

15. K. Deckers and A. Bultheel. "Recurrence and asymptotics for orthogonal rational functions on an interval", IMA Journal of Numerical Analysis 29(1):1-23, 2009.

16. K. Deckers, J. Van Deun, and A. Bultheel. "An extended relation between orthogonal rational functions on the unit circle and the interval [-1, 1]", Journal of Mathematical Analysis and Applications 334(2):1260-1275, 2007.

17. K. Deckers, J. Van Deun, and A. Bultheel. "Computing rational Gauss-Chebyshev quadrature formulas with complex poles: The algorithm", Advances in Engineering Software 40(8):707-717, 2009.

18. K. Deckers, J. Van Deun, and A. Bultheel. "Rational Gauss-Chebyshev quadrature formulas for complex poles outside [-1,1]", Mathematics of Computation 77(262):967983, 2008.

19. M.M. Džrbašian. "A survey on the theory of orthogonal systems and some open problems", in Orthogonal polynomials: Theory and practice, P. Nevai, editor, volume 294 of NATO-ASI Series C: Mathematical and Physical Sciences, pp. 135-146, Kluwer Academic Publishers, Boston, 1990.

20. B. Fritzsche, B. Kirstein, and A. Lasarow. "Orthogonal rational matrix-valued functions on the unit circle", Mathematische Nachrichten 278(5):525-553, 2005.

21. B. Fritzsche, B. Kirstein, and A. Lasarow. "Orthogonal rational matrix-valued functions on the unit circle: Recurrence relations and a Favard-type theorem", Mathematische Nachrichten 279(5-6):513-542, 2006.

22. W. Gautschi, L. Gori, and M.L. Lo Cascio. "Quadrature rules for rational functions", Numerische Mathematik 86(4):617-633, 2000.

23. L.B. Golinskii. "Quadrature formula and zeros of para-orthogonal polynomials on the unit circle", Acta Mathematica Hungarica 96(3):169-186, 2002.

24. W.B. Jones, O. Njåstad, and W.J. Thron. "Moment theory, orthogonal polynomials, quadrature, and continued fractions associated with the unit circle", Bulletin of the London Mathematical Society 21:113-152, 1989.

25. K. Müller and A. Bultheel. "Translation of the Russian paper "Orthogonal systems of rational functions on the unit circle" by M.M. Džrbašian", Technical Report TW253, Department of Computer Science, K.U.Leuven, February 1997. 
26. M. Riesz. "Sur le problème des moments, troisième note", Arkiv for Matematik, Astronomi och Fysik 17, no. 16, 52 pp., 1923.

27. B. Simon. Orthogonal Polynomials on the Unit Circle, Part 1: Classical Theory, AMS Colloquium Series, American Mathematical Society, Providence, RI, 2005.

28. B. Simon. Orthogonal Polynomials on the Unit Circle, Part 2: Spectral Theory, AMS Colloquium Series, American Mathematical Society, Providence, RI, 2005.

29. B. Simon. "Rank one perturbations and the zeros of paraorthogonal polynomials on the unit circle", Journal of Mathematical Analysis and Applications 329(1):376-382, 2007.

30. T.W. Tee and L.N. Trefethen. "A rational spectral collocation method with adaptively transformed Chebyshev grid points", SIAM Journal on Scientific Computing 28(5):1798-1811, 2006.

31. W. Van Assche and I. Vanherwegen. "Quadrature formulas based on rational interpolation", Mathematics of Computation 61(204):765-783, 1993.

32. J. Van Deun. "Eigenvalue problems to compute almost optimal points for rational interpolation with prescribed poles", Numerical Algorithms 45(1-4):89-99, 2007.

33. J. Van Deun and A. Bultheel. "A quadrature formula based on Chebyshev rational functions", IMA Journal of Numerical Analysis 26(4):641-656, 2006.

34. J. Van Deun and A. Bultheel. "An interpolation algorithm for orthogonal rational functions", Journal of Computational and Applied Mathematics 164/165:749-762, 2004.

35. J. Van Deun and A. Bultheel. "Computing orthonormal rational functions on a halfline", Rendiconti del Circolo di Palermo Serie II, Proceedings of the 5th International Conference on Functional Analysis and Approximation Theory (FAAT), Maratea, 16-22 June, 76:621-634, 2005.

36. J. Van Deun and A. Bultheel. "Modified moments and orthogonal rational functions", Applied Numerical Analysis and Computational Mathematics 1(2):455-468, 2004.

37. J. Van Deun and A. Bultheel. "Orthogonal rational functions and quadrature on an interval", Journal of Computational and Applied Mathematics 153(1-2):487-495, 2003.

38. J. Van Deun, A. Bultheel, and P. González-Vera. "Computing orthogonal rational functions with poles near the boundary", Computers and Mathematics with Applications 53(9):1421-1428, 2007.

39. J. Van Deun, A. Bultheel, and P. González-Vera. "On computing rational GaussChebyshev quadrature formulas", Mathematics of Computation 75(253):307-326, 2006.

40. J. Van Deun, K. Deckers, A. Bultheel, and J.A.C. Weideman. "Algorithm 882: Near best fixed pole rational interpolation with applications in spectral methods", $A C M$ Transactions on Mathematical Software 35(2):14:1-14:21, 2008.

41. P. Van gucht and A. Bultheel. "Using orthogonal rational functions for system identification", IEEE Transactions on Automatic Control 48(4):705-709, 2003.

42. J.A.C. Weideman. "Spectral methods based on nonclassical orthogonal polynomials", in Applications and computation of orthogonal polynomials (Oberwolfach, 1998), volume 131 of International Series of Numerical Mathematics, pp. 239251, Birkhäuser, Basel, 1999.

43. J.A.C. Weideman and D.P. Laurie. "Quadrature rules based on partial fraction expansions", Numerical Algorithms 24:159-178, 2000. 